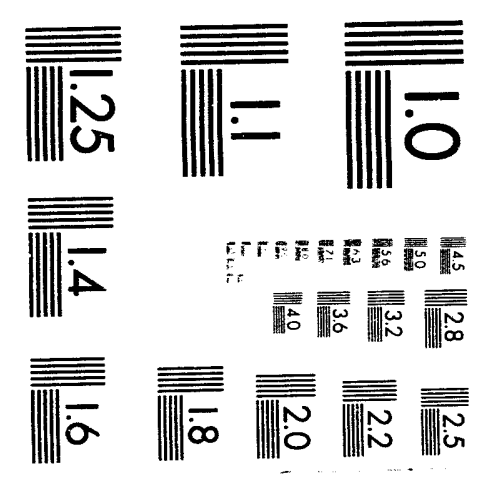



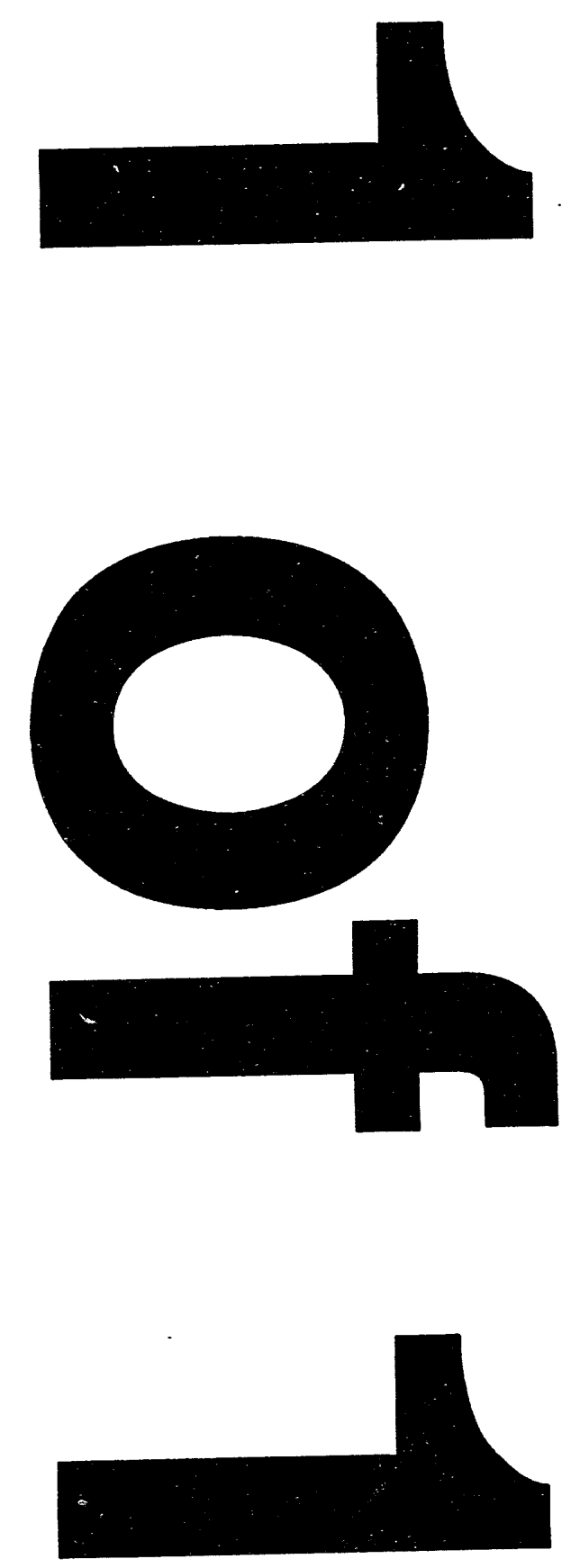


\title{
Bootstrap Current in a Tokamak
}

\author{
C. E. Kessel \\ Princeton Plasma Physics Laboratory, Princeton University \\ P. O. Box 451 \\ Princeton, New Jersey 08543
}

\begin{abstract}
The bootstrap current in a tokamak is examined by implementing the Hirshman-Sigmar model and comparing the predicted current profiles with those from two popular approximations. The dependences of the bootstrap current profile on the plasma properties are illustrated. The implications for steady state tokamaks are presented through two constraints; the pressure profle must be peaked and $\beta_{p}$ must be kept below a critical value.
\end{abstract}




\section{INTRODUCTION}

The bootstrap current was initially predicted by Bickerton, Connor, and Taylor[1] and has received considerable interest since the conclusion in TFTR[2] that the loop voltage measurements in their high beta-poloidal, neutral beam heated discharges could only be explained by including bootstrap current. Bootstrap currents have also been observed on JET[3] and JT-60[4]. It is now recognized that the bootstrap current is essential for steady state tokarnak reactors. Alternative methods for non-inductive current drive have very low efficiencies, so that providing $100 \%$ of the plasma current with these techniques would be prohibitively expensive. However, if the bootstrap current can provide $50 \%$ to $90 \%$ of the plasma current, the remainder can be provided by external sources to produce a viable steady state reactor concept.

A great deal of theoretical work has been done in deriving expressions for the bootstrap current within the scope of transport in toroidal plasmas ([5]-[11]). The most complete treatment for tokamaks (axisymmetric configurations) is that of Hirshman, et al.([12][17]), and subsequently the review paper by Hirshman and Sigmar[18]. More recently, some work has been done to incorporate simple expressions for the bootstrap current in equilibrium, transport, and systems studies([19]-[23]). A very interesting approach was given by Lackner[24] where he calculated nigh-n ballooning stability simultaneously with bootstrap current, albeit in the large aspect ratio approximation.

The present studies of bootstrap current have been motivated by the ARIES reactor study[25], particularly by second stability configurations where a large fraction of the plasma current is provided by the bootstrap effect. In this case we are interested in accurate predictions of the bootstrap current profile, how it can be maximized, how it can be aligned with a desired current profile (compatible from MHD stability), and how it limits the operating space for a tokamak reactor. In addition, a number of issues have arisen concerning how the bootstrap current is calculated; calculation of the trapped particle fraction, determination of the total toroidal current driven by the bootstrap effect, and the differences in predictions between several popular bootstrap models which make use of different approximations.

The fixed boundary, flux-coordinate equilibrium code JSOLVER[26] is used to generate the equilibrium and calculate the bootstrap current. The code can determine the bootstrap current for three models presently in use; collisionless Hirshman[20], collisional Harris[22], and the Hirshman-Sigmar[18] formulation. Only thermal species are considered here, although recent articles[27,28] have addressed the alpha particle contribution. There have also been extensions to nonaxisymmetric configurations[29], however, only the axisymmetric case is considered here.

A particular configuration for the plasma equilibria will be used with plasma major radius $R=5.6 \mathrm{~m}$, minor radius $a=1.4 \mathrm{~m}$, elongation $\kappa=1.8$, triangularity $\delta=0.4$, vacuum toroidal field $B_{\phi}=8.0 \mathrm{~T}$ (at $R=5.6 \mathrm{~m}$ ), plasma current $I_{p}=6.5 \mathrm{MA}$, and central safety factor $q_{0}=2.0$, which corresponds to the ARIES-II reactor design parameters. An impurity is included in the bootstrap calculations corresponding to $2 \%$ carbon, which gives an average charge $\bar{Z}=1.10$ and an effective charge $Z_{\text {eff }}=1.55$. The parallel current, 
pressure, and temperature profiles are taken to be of the form

$$
\begin{gathered}
\frac{\langle\vec{j} \cdot \vec{B}\rangle}{\langle\vec{B} \cdot \nabla \phi\rangle}(\psi)=j_{o}(1-\hat{\psi})^{a_{j}}, \\
p(\psi)=p_{o}\left(1-\hat{\psi}^{b_{p}}\right)^{a_{p}}, \\
T(\psi)=T_{o}\left(1-\hat{\psi}^{b_{T}}\right)^{a_{T}} .
\end{gathered}
$$

Here the normalized poloidal flux is $\hat{\psi}=\left(\psi-\psi_{\text {axis }}\right) /\left(\psi_{\text {lim }}-\psi_{\text {axis }}\right)$. The bootstrap calculations presented here are generally valid, and are not restricted to these profile forms. Table 1 will show the bootstrap current fractions and equilibrium profile parameters for each bootstrap current comparison.

\section{OVERVIEW OF THE BOOTSTRAP CURRENT}

The bootstrap current arises due to anisotropy in the electron pressure tensor. This is a neoclassical (toroidal geometry) effect which is important in the weakly collisional regimes (banana and plateau). This effect is due to the diffusion of particles that are barely trapped and barely circulating, and is strongly reduced when collisions impede the formation of banana orbits. The pressure anisotropy for the electrons leads to tangential forces within a magnetic flux surface. In steady state these forces are balanced by friction forces. When the momentum transfer between trapped and passing electrons is balanced with that between passing electrons and ions, currents are generated by the passing electrons, and this resulting current is the bootstrap current.

The trapped particle fraction is determined by the magnetic field structure in the tokamak. Charged particles gyrate around magnetic field lines, the center of this gyration being called the guiding center. For the majority of particles, this guiding center follows the helical magnetic field lines that trace out magnetic flux surfaces. However, since the magnetic field strength is not uniform on a magnetic surface, it follows from conservation of energy and magnetic moment that a certain fraction of particles will be reflected or "mirrored" when they enter a region of larger magnetic field. This causes them to become trapped, and to a first approximation they bounce back and forth between turning points along the magnetic field line[30]. In the large aspect ratio, circular cross-section, and low press'are approximation, the trapped particle fraction is given by

$$
f_{t} \approx 1.46 \sqrt{\epsilon}
$$

which shows the toroidal geometry dependence through the inverse aspect ratio $(\epsilon=r / R$, where $r$ is the local minor radius of the flux surface). The larger the trapped particle fraction, the larger the bootstrap current.

The collisionality is important in determining whether the bootstrap current is significant. When the plasma particles are highly collisional, even those that would normally be mirrored cannot execute trapped orbits before beino scattered, and the neoclassical effect is eliminated. Only when the collisionality is small is the bootstrap current important. The collisionality is normally represented by the ratio of the bounce frequency for 
a trapped particle to the frequency of collisions leading to a $90^{\circ}$ deflection in momentum space.

$$
\nu_{*}=\frac{q R}{v_{t h} \tau_{90} \epsilon^{\frac{3}{2}}}
$$

where $q$ is the safety factor for that flux surface, $R$ is the major radius of the flux surface, $v_{t h}$ is the thermal speed $(\sqrt{2 T / m})$, and $\tau_{90}$ is the $90^{\circ}$ deflection time. There are three main collisionality regimes; banana (collisionless), plateau (transition), and Phirsch-Schluter (collisional), and these are roughly delineated by the following relations,

$$
\begin{gathered}
\nu_{*} \ll 1 \quad(\text { banana }) \\
1 \leq \nu_{*} \leq \frac{1}{\epsilon^{\frac{3}{2}}} \quad(\text { plateau }) \\
\nu_{*} \gg 1 \quad(\text { collisional })
\end{gathered}
$$

The bootstrap current is diminished as the plasma becomes more collisional ( $\nu_{*}$ increases). However, the plateau regime can sustain a large bootstrap current and strongly modulate the current profile, and should not be considered a collisional regime for bootstrap current calculations.

\section{EXPRESSIONS FOR THE BOOTSTRAP CURRENT IN A TOKAMAK}

\subsection{Matrix Hirshman-Sigmar Model}

The bootstrap curren: is defined as a parallel current density, by the sum over all species of the product of dens ty, charge, and the parallel fluid flow $\left\langle u_{\|} B\right\rangle$,

$$
\langle\vec{j} \cdot \vec{B}\rangle_{b s}=\sum_{a} n_{a} Z_{a}\left\langle u_{a \|} B\right\rangle
$$

The parallel fluid and heat flows can be determined in terms of the thermodynamic flows by solving the parallel momentum and heat flux balance equations for each species. This derivation is done in Appendix A. This yields two equations for each specie which form a system that can be solved to determine the parallel fluid flows. This system of equations can be cast in a vector-matrix form by defining the vectors,

$$
\left[\left\langle u_{a \|} B\right\rangle,\left\langle u_{b||} B\right\rangle, \ldots, \frac{2}{5}\left\langle\frac{q_{a \|}}{p_{a}} B\right\rangle, \frac{2}{5}\left\langle\frac{q_{b \|}}{p_{b}} B\right\rangle, \ldots\right]^{T}
$$

and

$$
\left[V_{a 1}, V_{b 1}, \ldots, V_{a 2}, V_{b 2}, \ldots\right]^{T} .
$$

Here $V_{1}$ and $V_{2}$ are the thermodynamic flows defined in Appendix $\mathrm{A}$, and the subscripts "a" and " $b$ " refer to species. In order to illustrate the application of this formalism, the electron and single ion plasma will be used. Using the above defined vectors, the system 
of equations relating the parallel fluid and heat flows to the thermodynamic flows is given by

$$
\begin{gathered}
{\left[\begin{array}{cccc}
-l_{11}^{e e}+\bar{\mu}_{e 1} & -l_{11}^{e i} & l_{12}^{e e}+\bar{\mu}_{e 2} & l_{12}^{e i} \\
l_{11}^{i e} & -l_{11}^{i i}+\bar{\mu}_{i 1} & l_{12}^{i e} & l_{12}^{i i}+\bar{\mu}_{i 2} \\
l_{21}^{e \epsilon}+\bar{\mu}_{e 2} & l_{21}^{e^{i}} & -l_{22}^{e e}+\bar{\mu}_{e 3} & -l_{22}^{e_{2}^{i}} \\
l_{21}^{i e} & l_{21}^{i i}+\bar{\mu}_{i 2} & -l_{22}^{i e} & -l_{22}^{i i}+\bar{\mu}_{i 3}
\end{array}\right]\left[\begin{array}{l}
\left\langle u_{e \|} B\right\rangle \\
\left\langle u_{i \|} B\right\rangle \\
\frac{2}{5}\left\langle\frac{q_{e \|}}{p_{e}} B\right\rangle \\
\frac{2}{5}\left\langle\frac{q_{i \|}}{p_{e}} B\right\rangle
\end{array}\right]} \\
= \\
=\left[\begin{array}{cccc}
\bar{\mu}_{e 1} & 0 & \bar{\mu}_{e 2} & 0 \\
0 & \bar{\mu}_{i 1} & 0 & \bar{\mu}_{i 2} \\
\bar{\mu}_{e 2} & 0 & \bar{\mu}_{e 3} & 0 \\
0 & \bar{\mu}_{i 2} & 0 & \bar{\mu}_{i 3}
\end{array}\right]\left[\begin{array}{l}
V_{e 1} B \\
V_{i 1} B \\
V_{e 2} B \\
V_{i 2} B
\end{array}\right] .
\end{gathered}
$$

The first matrix can be inverted on the RHS to give the parallel fluid flows that are required to determine the parallel bootstrap current density. If the plasma contains $\mathrm{N}$ species ( $\mathrm{N}-1$ ion species and electrons) there are $2 \mathrm{~N}$ equations. It is clear how additional species can be added to the above formalism. The problem is reduced to determining the appropriate viscosity and friction coefficients to insert into the matrices. This model will be referred to as the Hirshman-Sigmar model. From Appendix A it can be seen that the parallel fluid flow depends on the pressure and temperature profiles, the collisionality, ion charges, aspect ratio, and trapped particle fraction.

$$
\left\langle u_{\|} B\right\rangle=f\left(p(\psi), T(\psi), \nu_{*}, Z, \epsilon, f_{t}\right) .
$$

The interactions between different species are manifested through the friction coefficients. These coefficients are classical, that is they are independent of the magnetic field. Expressions for these, which are valid for all neoclassical collisionality regimes, are given in Appendix C. In contrast, the viscosity coefficients are determined by the collisionality of the given species only, independent of the collisionality of all other species. They are also sensitive functions of the magnetic field structure. Expressions for the viscosity coefficients are given in Appendix B.

\subsection{Single Ion, Collisionless Regime Model}

The full matrix derivation given above is the most general treatment for determining the bootstrap current density due to thermal species, and can be extended to include as many species as required. A useful expression that is often used instead of the full matrix form is the single ion model in the collisionless $\left(\nu_{*} \rightarrow 0\right)$ limit. It is derived[20] for a plasma composed of electrons and a single ion species, with charge $Z_{i}$. Rather than using the full matrix formula, it starts from the balance equations for electrons and ions separately in vector form. This system of equations can be solved analytically because the matrices are $2 \times 2$. Terms of order $\left(m_{e} / m_{i}\right)^{\frac{1}{2}}$ or less are neglected, and $x$ is the ratio of trapped to circulating particles $\left(f_{t} /\left(1-f_{t}\right)\right)$. Using the quasi-neutrality condition $\left(n_{e}=Z_{i} n_{i}\right)$ the bootstrap current as a function of the thermodynamic flows, for a single ion plasma, is

$$
\frac{\langle\vec{j} \cdot \vec{B}\rangle_{b s}}{\langle\vec{B} \cdot \nabla \phi\rangle}=-\frac{p_{e}}{\left\langle\frac{1}{R^{2}}\right\rangle}\left[A_{1}\left[\frac{1}{p_{e}} \frac{d p_{e}}{d \psi}+\frac{p_{i}}{p_{e}}\left(\frac{1}{p_{i}} \frac{d p_{i}}{d \psi}-\alpha_{i} \frac{1}{T_{i}} \frac{d T_{i}}{d \psi}\right)\right]-A_{2} \frac{1}{T_{e}} \frac{d T_{e}}{d \psi}\right]
$$

with

$$
A_{1}=x\left(0.754+2.21 Z_{i}+Z_{i}^{2}\right)+x^{2}\left(0.347+1.24 Z_{i}+Z_{i}^{2}\right) / D_{e}
$$




$$
\begin{gathered}
A_{2}=x\left(0.885+2.08 Z_{i}\right) / D_{e} \\
D_{e}=\left[1.41 Z_{i}+Z_{i}^{2}+x\left(0.754+2.65 Z_{i}+2.00 Z_{i}^{2}\right)+x^{2}\left(0.347+1.24 Z_{i}^{2}+Z_{i}^{2}\right)\right] \\
\alpha_{i}=\frac{1.17}{1.0+0.46 x} .
\end{gathered}
$$

This model will be referred to as the Hirshman model.

\subsection{Single Ion, Harris Collisional Model}

In order to maintain the simplicity of the collisionless single ion expression for the bootstrap current but include collisional effects, Harris[22] presented a model that smoothly connects the collisionless Hirshman[20] expression with the collisional model of Hinton and Hazeltine[8]. The expression for the bootstrap current is nearly identical to that for the collisionless plasma, but the coefficients multiplying the gradients in pressure and temperature are modified to include factors that depend on $\nu_{*}$. The expression for the bootstrap current density in the Harris approximation is given by

$$
\frac{\langle\vec{j} \cdot \vec{B}\rangle_{b s}}{\langle\vec{B} \cdot \nabla \phi\rangle}=-\frac{p_{e}}{\left\langle\frac{1}{R^{2}}\right\rangle}\left[A_{1}^{H}\left[\frac{1}{p_{e}} \frac{d p_{e}}{d \psi}+\frac{p_{i}}{p_{e}}\left(\frac{1}{p_{i}} \frac{d p_{i}}{d \psi}-\alpha_{i}^{H} \frac{1}{T_{i}} \frac{d T_{i}}{d \psi}\right)\right]-\left(\frac{5}{2} A_{1}^{H}-A_{2}^{H}\right) \frac{1}{T_{e}} \frac{d T_{e}}{d \psi}\right]
$$

where,

$$
\begin{aligned}
A_{1}^{H} & =A_{1} \frac{1}{\left(1.0+a_{13} \nu_{* e}^{0.5}+b_{13} \nu_{* e}\right)} \frac{1}{\left(1.0+c_{13} \nu_{* e} \epsilon^{1.5}\right)} \\
A_{2}^{H} & =\left(\frac{5}{2} A_{1}-A_{2}\right) \frac{1}{\left(1.0+a_{23} \nu_{* e}^{0.5}+b_{23} \nu_{* e}\right)} \frac{1}{\left(1.0+c_{23} \nu_{* e} \epsilon^{1.5}\right)} \\
\alpha_{i}^{H} & =\left[\frac{\alpha_{i}-0.35 \nu_{* i}^{0.5}}{1.0+0.70 \nu_{* i}^{0.5}}-2.10 \nu_{* i}^{2} \epsilon^{3}\right] \frac{1}{\left(1.0+\nu_{* i}^{2} \epsilon^{3}\right)} \frac{1}{\left(1.0+\nu_{* e}^{2} \epsilon^{3}\right)}
\end{aligned}
$$

The coefficients $a_{i j}, b_{i j}$, and $c_{i j}$ are given in Ref[22] and the Hirshman collisionless coefficients are given in the preceeding section. It should be noted that the collisionalities used in the Harris expression are those originally defined by Hinton and Hazeltine in Ref[8]. This model will be referred to as the Harris model.

\subsection{Comparison of Bootstrap Current Models}

There are several characteristics that separate the various bootstrap models: matrix or single ion, collisionless or collisional, arbitrary aspect ratio or large aspect ratio, and the expression for the trapped particle fraction. The expression for the bootstrap current given by the Hirshman-Sigmar model is in the matrix form and can accomodate any number of species. The expressions for the Harris and Hirshman models are single ion forms, and only accomodate one ion specie. This ion is usually given the average characteristics of all the actual ions present. For example, the $Z_{i}$ in the expressions for $A_{1}$ and $A_{2}$ is made $Z_{\text {eff }}$ when multiple ions are present. However, there is some error in doing this, since the interaction between the different ion species is being neglected. Thus one would expect some differences between the single ion and matrix formulations when multiple ions are being modelled. 
It is generally clear whether a bootstrap model is collisionless or collisional, however the degree to which the three collisionality regimes are treated is what separates the various collisional models. The Hirshman model is strictly valid only in the collisionless regime. Refs. $[8,16,17,22]$ are examples of smooth fits for transport or viscosity coefficients over all collisionality regimes by connecting expressions derived asymptotically in each regime. The Harris model is an example of this, smoothly connecting the Hirshman collisionless expressions with the Hinton-Hazeltine collisional expressions for the bootstrap coefficients, $A_{1}$ and $A_{2}$. Efforts to generate expressions for these coefficients throughout all collisionality regimes by energy partitioning are reported in refs. $[14,15,17,31]$. The HirshmanSigmar model is an example of this approach. The differences among these models can lead to discrepancies, particularly in transitions between collisionality regimes. In addition, it is very difficult to isolate the cause of the discrepancies among the different models or which model is more or less correct, making comparisons confusing. The approach used in the Hirshman-Sigmar model[17] is the most general, and calculates expressions for the viscosity coefficients that are continuously valid throughout the three collisonality regimes and peforms the actual velocity space integrals. This is described in Appendix B.

The aspect ratio assumptions are also important in bootstrap current calculations. The Hirshman and Hirshman-Sigmar models are valid for arbitrary aspect ratio and flux surface geometry. The Harris model, as stated above, smoothly connects two models to create an expression that can be used for all three collisionality regimes. However, the collisionless model is valid for arbitrary flux surface shape and aspect ratio, while the collisional model was derived for circular flux surfaces and large aspect ratio. These inconsistencies can also lead to discrepancies among models and incorrect estimates of the bootstrap current. It should be emphasized that the collisionality changes across the plasma, so that the effects described here do not only influence the total bootstrap current but also its profile.

The model used for the trapped particle fraction can also have a significant effect on the predictions of a given bootstrap model. This is examined in Section 4.

The three models are compared with an equilibrium at high pressure where the bootstrap current is close to $100 \%$ of the equilibrium current, and are shown in Fig.(1). All use the full integral expression for the trapped particle fraction. The Hirshman-Sigmar model is considered the most accurate avail. ble for thermal species. The other two models are those described in the previous section, the Hirshman and Harris models. The peak temperature is $30 \mathrm{keV}$ for the first comparison, while the others are 15 and $7.5 \mathrm{keV}$, respectively. Differences in the Hirshman-Sigmar and Harris models are apparent, the former predicting more bootstrap current. The collisional models can be contrasted with the collisionless model by the absense of bootstrap current at the plasma edge. The underprediction of bootstrap current for the Harris model is evident here, particularly as the plasma becomes more collisional (temperature is lowered). The discrepancy between the Hirshman-Sigmar and Harris models at higher temperature is mostly due to the single ion approximation. As the temperature is lowered, the approximate model for collisionality in the later is responsible. The resulting bootstrap fractions for the different cases are given in Table 1 for the three temperatures and the various models. 


\section{TRAPPED PARTICLE FRACTION MODELS}

There are several models for the trapped particle fraction, primarily because the full two-dimensional integral is complicated to evaluate. The actual expression is given by[18]

$$
f_{t}=1-\frac{3}{4}\left\langle B^{2}\right\rangle \int_{0}^{\frac{1}{B_{\max }}} \frac{\lambda d \lambda}{\langle\sqrt{1-\lambda \bar{B}}\rangle}
$$

where $\lambda=1 / B$ and $B_{\max }$ refers to the maximum value of $B$ on a flux surface (assuming only a single maximum), and the angular brackets refer to the flux surface average. A common approximation is to assume the variation of the magnetic field around a flux surface, typically the large aspect ratio and low pressure expression,

$$
B=\frac{B_{o}}{\left(1+\frac{a}{R} \cos \theta\right)}
$$

The integral can be evaluated directly to give[19]

$$
f_{t}=1-\frac{(1-\epsilon)^{2}}{\sqrt{1-\epsilon^{2}}(1+1.46 \sqrt{\epsilon})} .
$$

However, since the magnetic field variation is assumed the expression can be in error for lower aspect ratios, shaped flux surfaces, and higher pressures.

Another approach to approximate the actual integral was taken in Ref[32], by replacing the integrand with $\lambda\left\langle(1-\lambda B)^{-\frac{1}{2}}\right\rangle$, and taking the surface average integration outside of the $\lambda$ integration. The integral can then be performed analytically to give

$$
f_{t}=1-\left\langle B^{2}\right\rangle\left\langle B^{-2}\right\rangle+\frac{3}{2}\left\langle B^{2}\right\rangle\left\langle B^{-2}\left[\left(1-\frac{B}{B_{\max }}\right)^{\frac{1}{2}}-\frac{1}{3}\left(1-\frac{B}{B_{\max }}\right)^{\frac{3}{2}}\right]\right\rangle .
$$

This expression involves the flux-surface average of the magnetic field and so also requires flux surface geometry. This is more accurate than Eqn.(13), and is faster to evaluate than the full integral.

To see the impact of the trapped particle fraction model on the calculation of the bootstrap current, the current profiles are shown in Fig.(2). The bootstrap current fractions using the three expressions are given in Table 1. This also illustrates the impact of the trapped particle expression on alignment of the bootstrap current with the equilibrium current profile.

\section{DETERMINING TOTAL TOROIDAL CURRENT FROM PARALLEL BOOTSTRAP CURRENT}

The expression for the bootstrap current density is given as a parallel current, while the total plasma current refers to toroidally directed current only. Thus the expression $\langle\vec{j} \cdot \vec{B}\rangle_{b s}$ must be converted to a toroidally directed current in order to compare it to the plasma current that is typically quoted.

The total toroidal current in the plasma can be written as

$$
I_{\phi}=\int_{A_{p}} d R d Z j_{\phi}(R, Z)=\int_{A_{p}} d \psi \frac{d l_{\theta}}{R B_{\theta}} j_{\phi}(R, \psi) .
$$


Here $d l_{\theta}$ is the arc length in the poloidal direction along a flux surface and $B_{\theta}$ is the poloidal magnetic field. Using the standard definition for the flux surface average of a quantity,

$$
\langle A\rangle=\frac{\int \frac{d l_{\theta}}{B_{\theta}} A}{\int \frac{d l_{\theta}}{B_{\theta}}}
$$

Eqn.(15) can be rewritten as

$$
I_{\phi}=\int_{A_{p}} d \psi\left\langle\frac{j_{\phi}}{R}\right\rangle \int \frac{d l_{\theta}}{B_{\theta}}
$$

The goal is then to get an expression for $\left\langle j_{\phi} / R\right\rangle$ in terms of the parallel current density $\langle\vec{j} \cdot \vec{B}\rangle /\langle\vec{B} \cdot \nabla \phi\rangle$. Using Eqn.(29) for the magnetic field in a tokamak, the corresponding toroidal current density is,

$$
j_{\phi}=-R \frac{d p}{d \psi}-\frac{1}{2 \mu_{o} R} \frac{d g^{2}}{d \psi}
$$

The expression for $\mu_{o} \vec{j} \cdot \vec{B}$ can be written as

$$
\mu_{o} \vec{j} \cdot \vec{B}=-\frac{d g}{d \psi} B_{\theta}^{2}-\mu_{o} g \frac{d p}{d \psi}-\frac{1}{2} \frac{g}{R^{2}} \frac{d g^{2}}{d \psi} .
$$

After dividing by $\langle\vec{B} \cdot \nabla \phi\rangle=\left\langle B_{\phi} / R\right\rangle=g\left\langle 1 / R^{2}\right\rangle$ we get,

$$
\mu_{\circ} \frac{\vec{j} \cdot \vec{B}}{\langle\vec{B} \cdot \nabla \phi\rangle}=-\frac{1}{2} \frac{B_{\theta}^{2}}{\left\langle B_{\phi}^{2}\right\rangle} \frac{d g^{2}}{d \psi}-\mu_{o} \frac{1}{\left\langle\frac{1}{R^{2}}\right\rangle} \frac{d p}{d \psi}-\frac{1}{2} \frac{B_{\phi}^{2}}{\left\langle B_{\phi}^{2}\right\rangle} \frac{d g^{2}}{d \psi} .
$$

It follows after flux surface averaging,

$$
\frac{1}{2} \frac{d g^{2}}{d \psi}=\frac{\left\langle B_{\phi}^{2}\right\rangle}{\left\langle B^{2}\right\rangle}\left[-\mu_{o} \frac{\langle\vec{j} \cdot \vec{B}\rangle}{\langle\vec{B} \cdot \nabla \phi\rangle}-\mu_{o} \frac{1}{\left\langle\frac{1}{R^{2}}\right\rangle} \frac{d p}{d \psi}\right] .
$$

This expression can now be substituted into Eqn.(18). Rearranging, dividing by $R$, and taking the flux surface average gives,

$$
\left\langle\frac{j_{\phi}}{R}\right\rangle=-\frac{d p}{d \psi}\left(1-\frac{\left\langle B_{\phi}^{2}\right\rangle}{\left\langle B^{2}\right\rangle}\right)+\left\langle\frac{1}{R^{2}}\right\rangle \frac{\langle\vec{j} \cdot \vec{B}\rangle}{\langle\vec{B} \cdot \nabla \phi\rangle} \frac{\left\langle B_{\phi}^{2}\right\rangle}{\left\langle B^{2}\right\rangle} .
$$

The first term represents the contribution from perpendicular current, that is the toroidal component of the Pfirsch-Schluter current. Since the bootstrap current density is a parallel current, only the second term is kept for determining a total bootstrap current, giving

$$
I_{\phi}^{b s}=\int d \psi\left[\langle\vec{B} \cdot \nabla \phi\rangle \frac{\langle\vec{j} \cdot \vec{B}\rangle_{b s}}{\left\langle B^{2}\right\rangle} \int \frac{d l}{B_{\theta}}\right]=\int d \psi \frac{q(\psi)}{\left\langle B^{2}\right\rangle}\langle\vec{j} \cdot \vec{B}\rangle_{b s},
$$

where $q(\psi)$ is the safety factor. 


\section{DEPENDENCES OF THE BOOTSTRAP CURRENT}

A simple global representation for the ratio of bootstrap current to total plasma current is obtained by inserting Eqn.(9) into Eqn.(23) and expressing the result in the form,

$$
\frac{I_{b s}}{I_{p}}=C_{b s} \sqrt{\epsilon} \beta_{p}
$$

where $C_{b s}$ is a function of several quantities; density, temperature, and current profiles, ion charge, collisionality, trapped particle fraction, and the aspect ratio (for higher order terms). As $\beta_{p}$ is increased the bootstrap current ratio will increase. Fig.(3) shows the bootstrap current profiles for $\beta_{p}$ values of $1.0,2.0,3.0$, and 4.0. The corresponding bootstrap fractions are given in Table 1 . As can be seen in the last case, if $\beta_{p}$ is high enough, one can generate more bootstrap current than the desired total plasma current. The variations for the same value of $\beta_{p}$ that are possible, due to the hidden dependences in $C_{b s}$, are quite large. This is discussed in the context of zero-dimensional calculations in a recent paper[23]. The dependence of the bootstrap current ratio on inverse aspect ratio can only be understood by replacing the product $\sqrt{\epsilon} \beta_{p}$ by $\epsilon \beta_{p} / \sqrt{\epsilon}$. This is necessary because $\epsilon \beta_{p}$ is the appropriate quantity to hold constant when making a comparison at different aspect ratios. It is then clear that larger bootstrap current fractions will be obtained at higher aspect ratio. Since the bootstrap current profile and its alignment with a desired equilibrium current profile are of interest, the additional dependences will be discussed in terms of full profiles, rather than a zero-dimensional model. The most important dependences are the kinetic profiles, the collisionality, and the trapped particle fraction.

A rough representation of the bootstrap current density for large aspect ratio and low pressure, in the collisionless limit can be obtained by using Eqn.(9) to solve for the parallel current density

$$
j_{\|}^{b s} \approx-\sqrt{\epsilon} R\left[2.4 T \frac{d n}{d \psi}+0.1 n \frac{d T}{d \psi}\right] .
$$

This expression can be cast in another form by defining the parameter $\eta=n T^{\prime} / n^{\prime} T[24]$, where primes denote differentiation with respect to $\psi$.

$$
j_{\|}^{b s} \approx-\sqrt{\epsilon} R \frac{d p}{d \psi}\left[2.4\left(\frac{1}{1+\eta}\right)+0.1\left(\frac{\eta}{1+\eta}\right)\right]
$$

This expression can help to understand the profile dependences that will be discussed in the following.

The pressure profile (more specifically the pressure gradient) determines the overall shape of the bootstrap current profile; peaked pressure profiles cause the bootstrap current to peak near the plasma center, and broad pressure profiles cause the bootstrap current to peak near the plasma edge. This is illustrated in Fig.(4) for four different pressure profiles. This can be understood by examining Eqn.(26). The pressure gradient in the expression is dominant, while the term $\eta$ is more slowly varying for changes in $n(\psi)$ and $T(\psi)$. In fact, for profiles of the form $(1-\hat{\psi})^{a}, \eta$ becomes a constant and the shape of $j_{\|}^{b s}$ is determined solely by $d p / d \psi$. Table 1 gives the corresponding pressure and temperature profile parameters for each of these cases. 
The next most important profile is the density profile, which strongly modulates the overall magnitude of the bootstrap current. More peaked densities lead to larger bootstrap currents, and visa versa. This is illustrated in Fig.(5), where four different density profiles are used. The temperature profile is adjusted to keep the pressure profile fived. Table 1 gives the values for the peak to average of the density profiles and the corresponding bootstrap current fractions. The change in profile shape at the plasma edge is due to collisionality, which will be discussed next. The density profile effects can be seen by examining Eqn.(26). The first term in the square brackets provides for most of the bootstrap current. For the limiting cases of $n^{\prime}$ going to zero and infinity, the first term goes to zero and 2.4, respectively. Thus, having a higher density gradient leads to more bootstrap current, and visa versa. The second term in the square brackets contributes, but only significantly when the density gradient is small. The shape of the density profile can also influence the shape of the bootstrap current profile, but is a weaker effect than the pressure gradient.

The collisionality can have a strong impact on the magnitude and the shape of the bootstrap profile. Since the collisicnality scales as $p / T^{3}$, and the bootstrap current decreases as the collisionality increases, there is a strong temperature effect. This is illustrated in Fig.(6), where the peak temperature is lowered from 30 to 7.5 , and $3.75 \mathrm{keV}$. The profiles are held fixed, and the density is increased by the same factor that the temperature is reduced to keep the pressure fixed. The region at the plasma edge is more collisional since the temperature decreases there, and this region enlarges as the temperature is dropped. An interesting effect can be seen in the bootstrap current profile when the collisionality moves from the banana to the plateau regimes. The bootstrap current can actually becomes larger locally than the value for the banana regime. This appears counterintuitive, but arises from the temperature gradient terms. In the form for the bootstrap current presented here (for example Eqn.(9)), typically the pressure gradient terms contribute to the bootstrap current and the temperature gradient terms subtract from it. As the collisionality transitions from banana to plateau regimes, the coefficients multiplying the temperature gradient terms can locally become diminished, leading to a local increase in the bootstrap current. This effect is best illustrated in Figs.(1a) and (1b).

In summary, the primary plasma properties that impact the bootstrap current magnitude and its profile shape are (i) $\beta_{p}$ (or total pressure) which determines the bootstrap current magnitude, (ii) the plasma pressure profile which determines the bootstrap current shape, (iii) the plasma density profile that effects the bootstrap current magnitude and more weakly its shape, (iv) and collisionality (primarily plasma temperature and its profile) that influences the bootstrap current magnitude and shape.

\section{THE IMPLICATIONS OF BOOTSTRAP CURRENT}

The dependences described in the previous section put severe constraints on tokamaks in steady state, where the fully developed bootstrap current would be present. In steady state the plasma current will be composed of a bootstrap and an external current drive component. Non-inductive methods for external current drive have very low efficiencies. It is therefore advantageous to generate as much bootstrap current as possible and minimize the external drive component. However, as shown earlier in this section, the bootstrap 
current profile will depend on the plasma's density and temperature profiles. The resulting combination of current and pressure profiles must then be MHD stable. Both the profile of the bootstrap current and its magnitude are equally important.

It should be noted that most present day experiments do not have fully developed bootstrap currents because their discharge times are too short. The bootstrap current requires significant $\beta_{p}$ to be an important fraction of the total plasma current, and develops temporally in the plasma according to the local value of the plasma resistivity. Since the resistivity can be quite low in a hot plasma the bootstrap current can take a long time to appear. In addition, since the temperature normally decreases from the plasma center to the edge, the bootstrap current will appear at the plasina edge first and continue to develop toward the center. Thus, many plasma configurations generated in experimental tokamaks would not survive in steady state due to MHD unstable combinations of current and pressure profile:

The constraints imposed by the bootstrap effect on tokamak configurations are on the pressure profile for current profile alignment to keep $\langle\vec{j} \cdot \vec{B}\rangle_{b s} \leq\langle\vec{j} \cdot \vec{B}\rangle_{e q}$, and the achievable $\beta_{p}$, to keep $I_{b s} \leq I_{p}$. The bootstrap limit on $\beta_{p}$ can be more restrictive in limiting $\beta$ than MHD stability. This limit is primarily a function of the density profile. To achieve a bootstrap fraction near unity, $\beta_{p}$ can be larger for broad density profiles than for peaked density profiles. Since the plasma $\beta$ is proportional to $\beta_{p}$ this becomes a limit on the achievable pressure in the plasma. This leads to the conclusion that broad densities profiles can lead to larger values of accessible $\beta$, however, the extent of this benefit depends on the bootstrap current alignment.

The stability requirement for equilibria with significant bootstrap fractions leads to the concept of alignment. In general, there are desirable combinations of current and pressure profiles that yield ideal MHD stable equilibria. This alignment requires that the resulting bootstrap current profile be less than or equal to the given equilibrium current profile $\left(\langle\vec{j} \cdot \vec{B}\rangle_{b s} \leq\langle\vec{j} \cdot \vec{B}\rangle_{e q}\right)$ across the plasma. Where the bootstrap current is less, external current drive must make up the difference. In general, plasma pressure profiles must be relatively peaked to keep the bootstrap current near the plasma center, and to avoid large currents near the plasma edge which are known to be destabilizing to external kink modes. Although ideal MHD stability analysis([33]-[35]) indicates that broad pressure profiles can be stabilizing for low-n external kink modes, the bootstrap current that would result in steady state for these equilibria would be highly destabilizing. An interesting situation results when the equilibrium current profile is made to take on the shape of the bootstrap current profile. The bootstrap current profile is always hollow, producing safety factor profiles that have an off-axis minimum. Assuming there was some source of current at the plasma axis (seed current), one can produce stable equilibria with such current profiles[36]. An equilibrium of this type is illustrated in Fig.(7).

A technique we use to help find well aligned bootstrap current profiles is by inverting the collisionless bootstrap expression. The expression for the bootstrap current in the collisionless limit for the electron and single ion plasma is given by Eqn.(9). This equation depends on the temperature and pressure profiles, the ion charge, and the trapped particle fraction. However, this is independent of the collisionality, and therefore is independent of the actual temperature. Since the bootstrap current calculation is typically done after an equilibrium calculation, the profiles for the pressure $p(\psi)$ and the equilibrium current $\langle\vec{j} \cdot \vec{B}\rangle_{e q} /\langle\vec{B} \cdot \Gamma \phi\rangle$ are already specified. If we substitute the equilibrium current profile 
on the LHS and the pressure profile on the RHS, the equation can be inverted to give the normalized temperature gradient. If we assume the electron and ion temperature profiles are the same, this then gives the temperature profile that will cause the bootstrap current profile to align with the equilibrium current profile, for the specified pressure profile. Fig.(8) shows results for three cases where the temperature profile specified is made to agree fairly closely with the one derived by the inversion. One curve in the temperature and density plots are the derived profiles for alignment, while the other curves are the actual profiles used. The results will not be meaningful if the plasma does not possess enough pressure to obtain a ratio of bootstrap current to total plasma current of the order unity, or if the pressure profile shape cannot place the bootstrap current in the desired location. A bootstrap current model that includes collisionality would become temperature dependent, however, the collisionless result can still be quite useful in getting near alignment. For example, minor changes in the temperature and density profiles will yield better alignment for the cases shown in Fig.(8).

\section{CONCLUSIONS}

Since there are several different models of the bootstrap current commonly being used, the Hirshman-Sigmar model, which is considered the most accurate for thermal species, was implimented and compared to two other popular approximations, the collisionless Hirshman[20] and collisional Harris[22] models. The Hirshman-Sigmar model is a matrix method and can accomodate any number of species, while the other two models are single ion formulations, only accomodating electrons and a single "average" ion. The use of single ion formulas leads to some discrepancies because the interaction between ions is neglected. As the plasma becomes more collisional, the collisionless model is invalid, while the Harris model consistently under-predicts the bootstrap current as compared to the HirshmanSigmar model. This is attributed to the connection of two inconsistent models in the Harris formulation to construct a model that can be used in all collisionality regimes. Although the full Hirshman-Sigmar model is more complicated to evaluate, the author has sped up the velocity space integrals required for the evaluation of the viscosity coefficients, which makes the computational time only slightly longer than the other methods. The less accurate models for the bootstrap current should only be used when computational speed is critical.

A comparison of two trapped particle fraction approximations with the full integral expression shows that additional discrepancies in the bootstrap current will arise from using large aspect ratio expressions like Eqn.(13).

The dependences of the bootstrap current on the plasma properties have been illustrated, and can be used both for interpretation and development of tokamak configurations with significant bootstrap fractions. The bootstrap current magnitude and its profile are primarily determined by $\beta_{p}$ (or total pressure), the location of the pressure gradient, the peakedness of the density profile, and collisionality (through the temperature and its profile).

Since the bootstrap current has been experimentally observed, its implications for steady state or long pulse tokamaks are important. When examining tokamak configurations for ideal MHD stability and external current drive, the bootstrap current must be 
included self-consistently. There are combinations of pressure and current profiles that yield MHD stable equilibria, and the bootstrap current profile must align with an allowed current profile $\left(\langle\vec{j} \cdot \vec{B}\rangle_{b s} \leq\langle\vec{j} \cdot \vec{B}\rangle_{e q}\right)$ and at the same time be self-consistent with the pressure profile and $\beta_{p}$ that produce it. Any part of the equilibrium current profile not provided by the bootstrap current is presumed to be supplied by the external current drive. Due to the above requirements two constraints arise; the pressure profile must be relatively peaked to place the bootstrap current near the plasma center and avoid large currents near the plasma edge, and $\beta_{p}$ will be limited to lie below a critical value (for a given density profile) to keep the bootstrap current below the desired plasn:a current. 


\section{Appendix A: DERIVATION OF THE BOOTSTRAP CURRENT RELATION FROM PARALLEL BALANCE EQUATIONS}

The parallel momentum and heat flux balance equations for each species are given in Ref[16] as,

$$
\begin{aligned}
& \left\langle\vec{B} \cdot \nabla \cdot \tilde{\Pi}_{a}\right\rangle=\left\langle F_{a 1} B\right\rangle, \\
& \left\langle\vec{B} \cdot \nabla \cdot \tilde{\Theta}_{a}\right\rangle=\left\langle F_{a 2} B\right\rangle .
\end{aligned}
$$

Here $\tilde{\Pi}_{a}$ and $\tilde{\Theta}_{a}$ are the viscous and viscous heat stress tensors for species $a$, and $F_{a 1}$ and $F_{a 2}$ are the friction forces. The angular brackets refer to flux surface averages. The constant electric field that would appear in the first equation to represent ohmic current drive is neglected, since only the bootstrap current is of interest here. These balance equations apply on each flux surface in the plasma. Using the standard representation for the magnetic field in a tokamak, $\vec{B}$ is given by,

$$
\vec{B}=\nabla \phi \times \nabla \psi+g(\psi) \nabla \phi,
$$

where the toroidal field function is defined as $g(\psi)=R B_{\phi}$. The tensor quantities are defined in terms of poloidal fluid and heat flows $\left(u_{\theta}\right.$ and $\left.q_{\theta}\right)$ and viscosity coefficients $\mu_{1,2,3}$.

$$
\begin{aligned}
& \left\langle\vec{B} \cdot \nabla \cdot \tilde{\Pi}_{a}\right\rangle=3\left\langle(\hat{n} \cdot \nabla B)^{2}\right\rangle\left[\mu_{a 1} u_{a \theta}+\frac{2}{5} \mu_{a 2} \frac{q_{a \theta}}{p_{a}}\right] \\
& \left\langle\vec{B} \cdot \nabla \cdot \tilde{\Theta}_{a}\right\rangle=3\left\langle(\hat{n} \cdot \nabla B)^{2}\right\rangle\left[\mu_{a 2} u_{a \theta}+\frac{2}{5} \mu_{a 3} \frac{q_{a \theta}}{p_{a}}\right] .
\end{aligned}
$$

The unit vector $\hat{n}$ is parallel to the magnetic field and defined as $\vec{B} / B$. The viscosity coefficients are defined in Appendix B. Following Ref[16], we note that the first order neoclassical particle and heat flows are strictly within a flux surface, which allows a simple relation between the poloidal flows and parallel flows. The dot product of the poloidal magnetic field is taken with the total particle and heat flows $\left(\vec{u}_{a}=\vec{u}_{a \perp}+\vec{u}_{a \|}\right.$ and $\vec{q}_{a}=$ $\left.\vec{q}_{a \perp}+\vec{q}_{a \|}\right)$. The poloidal flows can then be related to the parallel flows by,

$$
\begin{gathered}
u_{a \|}=V_{a 1}+u_{a \theta} B \\
\frac{2}{5} \frac{q_{a \|}}{p_{a}}=V_{a 2}+\frac{2}{5} \frac{q_{a \theta}}{p_{a}} B .
\end{gathered}
$$

Here $V_{a 1}$ and $V_{a 2}$ are the parallel flows whose poloidal component is equal to the diamagnetic drift velocity, and are defined by

$$
\begin{gathered}
V_{a 1}=-\frac{R B_{\phi} T_{a}}{Z_{a} B}\left[\frac{1}{p_{a}} \frac{d p_{a}}{d \psi}+\frac{Z_{a}}{T_{a}} \frac{d \Phi}{d \psi}\right] \\
V_{a 2}=-\frac{R B_{\phi} T_{a}}{Z_{a} B}\left[\frac{1}{T_{a}} \frac{d T_{a}}{d \psi}\right]
\end{gathered}
$$

where $T_{a}$ is temperature, $Z_{a}$ is the charge of species $a$, and $B_{\phi}$ is the toroidal field. We will refer to these as thermodynamic flows. The electric potential $\Phi$ gives rise to $E \times B$ 
flow which is the same for all species and thus does not produce any friction force in the final expression.

The friction forces can also be related to the parallel flows in terms of the friction coefficients,

$$
\begin{gathered}
F_{a 1}=\sum_{b}\left(l_{11}^{a b} u_{b \|}-\frac{2}{5} l_{12}^{a b} \frac{q_{b \|}}{p_{b}}\right) \\
F_{a 2}=\sum_{b}\left(-l_{21}^{a b} u_{b \|}+\frac{2}{5} l_{22}^{a b} \frac{q_{b \|}}{p_{b}}\right)
\end{gathered}
$$

where $b$ represents all species, including species $a$, and the $l_{i j}$ are the friction coefficients, which are defined in Appendix C. There are additional terms called secondary flows[15] that would appear in the definition of the friction forces, however, they represent corrections of higher order and are neglected in the present work. Before substituting to get the parallel balance equations strictly in terms of parallel flows and thermodynamic flows, the product of $B$ is taken with Eqns.(32) and (33), and the flux surface average is taken. The viscosity coefficients are redefined to be,

$$
\bar{\mu}_{a i}=\frac{3\left\langle(\hat{n} \cdot \nabla B)^{2}\right\rangle}{\left\langle B^{2}\right\rangle} \mu_{a i} .
$$

Combining Eqns.(27-38) gives two equations for each specie in the plasma,

$$
\begin{gathered}
\bar{\mu}_{a 1}\left\langle u_{a||} B\right\rangle-\bar{\mu}_{a 1} V_{a 1} B+\frac{2}{5} \bar{\mu}_{a 2}\left\langle\frac{q_{a \|}}{p_{a}} B\right\rangle-\bar{\mu}_{a 2} V_{a 2} B=\sum_{b}\left(l_{11}^{a b}\left\langle u_{b \|} B\right\rangle-\frac{2}{5} l_{12}^{a b}\left\langle\frac{q_{b \|}}{p_{b}} B\right\rangle\right) \\
\bar{\mu}_{a 2}\left\langle u_{a||} B\right\rangle-\bar{\mu}_{a 2} V_{a 1} B+\frac{2}{5} \bar{\mu}_{a 3}\left\langle\frac{q_{a \|}}{p_{a}} B\right\rangle-\bar{\mu}_{a 3} V_{a 2} B=\sum_{b}\left(-l_{21}^{a b}\left\langle u_{b \|} B\right\rangle+\frac{2}{5} l_{22}^{a b}\left\langle\frac{q_{b \|}}{p_{b}} B\right\rangle\right) .
\end{gathered}
$$

These equations form the system to be solved for obtaining the parallel fluid and heat flows in terms of the thermodynamic flows. 


\section{Appendix B: VISCOSITY COEFFICIENTS}

The viscosity coefficients are defined with respect to viscosity matrix terms $K_{i j}^{a}$ by the following,

$$
\begin{gathered}
\bar{\mu}_{a 1}=K_{11}^{a} \\
\bar{\mu}_{a 2}=K_{12}^{a}-\frac{5}{2} K_{11}^{a} \\
\bar{\mu}_{a 3}=K_{22}^{a}-5 K_{12}^{a}+\frac{25}{4} K_{11}^{a} .
\end{gathered}
$$

The $a$ is the species index. In the Hirshman-Sigmar[18] review paper, the coefficients $K_{i j}^{a}$ are derived asymptotically in each collisionality regime; Phirsh-Schluter, plateau, and banana. Each of these results are strictly valid only in the respective regime. The paper then presents a prescription for the viscosity coefficients that is continuously valid throughout the three regimes. This approach is used here. The coefficients $K_{i j}^{a}$ are defined by

$$
K_{i j}^{a}=\frac{f_{t}}{f_{c}} \frac{n_{u} m_{a}}{\tau_{a a}}\left[x_{a}^{2(i+j-2)} \nu_{t o t}^{a} \tau_{a a}\right]
$$

where $f_{t}$ and $f_{c}$ are the trapped and circulating particle fractions, respectively. $n_{a}$ is the density, $m_{a}$ is the particle mass, and $\tau_{a a}$ is the self collision time for $90^{\circ}$ deflections in momentum space. The brackets refer to an integration of the enclosed quantity over velocity,

$$
[A(v)]=\int_{0}^{\infty} d x x^{4} e^{-x^{2}} A\left(x v_{t a}\right) .
$$

$x_{a}$ is the ratio of particle velocity to thermal velocity $\left(v / v_{t a}\right)$, where $v_{t a}=\sqrt{2 T_{a} / m_{a}}$. $\nu_{t o t}^{a}(v)$ is the total collision frequency and is defined by,

$$
\nu_{t o t}^{a}(v)=\frac{\nu_{D}^{a}(v)}{\left(1+2.48 \nu_{* a} \frac{\nu_{D}^{a}(v) \tau_{a a}}{x_{a}}\right)\left(1+1.96 \nu_{* a} \frac{\nu_{\perp}^{a}(v) \tau_{a a}}{x_{a}}\right)} .
$$

The various collision times and frequencies are defined below. The $90^{\circ}$ self scattering time is

$$
\tau_{a a}=\frac{12 \pi^{1.5} \epsilon_{o}^{2}}{4} \frac{m_{a}^{2} v_{t a}^{3}}{n_{a} Z_{a}^{4} e^{4} \ln \Lambda} .
$$

The $90^{\circ}$ scattering time of species $a$ from species $b$ is

$$
\tau_{a b}=\frac{12 \pi^{1.5} \epsilon_{o}^{2}}{4} \frac{m_{a}^{2} v_{t a}^{3}}{n_{b} Z_{b}^{2} Z_{a}^{2} e^{4} \ln \Lambda} .
$$

The collisionality parameter is given by

$$
\nu_{* a}=\frac{R q}{v_{t a} \tau_{a a} \epsilon^{\frac{3}{2}}} .
$$

The pitch angle scattering frequency is

$$
\nu_{D}^{a}(v)=\frac{3 \sqrt{\pi}}{4} \sum_{b} \nu_{D}^{a b}(v)=\frac{3 \sqrt{\pi}}{4} \frac{1}{\tau_{a a}} \sum_{b} \frac{n_{b} Z_{b}^{2}}{n_{a} Z_{a}^{2}}\left[\frac{\Phi\left(x_{b}\right)-G\left(x_{b}\right)}{x_{a}^{3}}\right] .
$$


The test particle scattering frequency is

$$
\nu_{t}^{a}(v)=3 \nu_{D}^{a}(v)+\nu_{E}^{a}(v) .
$$

The energy exchange frequency is

$$
\nu_{E}^{a}(v)=2 \nu_{s}^{a}(v)-2 \nu_{D}^{a}(v)-\nu_{p}^{a}(v) .
$$

The slowing down frequency is

$$
\nu_{s}^{a}(v)=\frac{3 \sqrt{\pi}}{4} \sum_{b} \nu_{s}^{a b}(v)=\frac{3 \sqrt{\pi}}{4} \frac{1}{\tau_{a a}} \sum_{b} \frac{n_{b} Z_{b}^{2}}{n_{a} Z_{a}^{2}}\left[2\left(\frac{T_{a}}{T_{b}}+\frac{v_{t a}^{2}}{v_{t b}^{2}}\right) \frac{G\left(x_{b}\right)}{x_{a}}\right] .
$$

The parallel velocity scattering frequency is

$$
\nu_{p}^{a}(v)=\frac{3 \sqrt{\pi}}{4} \sum_{b} \nu_{p}^{a b}(v)=\frac{3 \sqrt{\pi}}{4} \frac{1}{\tau_{a a}} \sum_{b} \frac{n_{b} Z_{b}^{2}}{n_{a} Z_{a}^{2}}\left[\frac{2 G\left(x_{b}\right)}{x_{a}^{3}}\right] .
$$

The functions $\Phi(x)$ and $G(x)$ are the error and Chandrasakar functions, respectively, defined by,

$$
\begin{gathered}
\Phi(x)=\frac{2}{\sqrt{\pi}} \int_{0}^{x} e^{-u^{2}} d u \\
G(x)=\frac{\Phi(x)-x \frac{d \Phi}{d x}}{2 x^{2}} .
\end{gathered}
$$

The coefficient 2.48 in the expression for $\nu_{t o t}^{a}(v)$ is a large aspect ratio approximation to that given in $\operatorname{Ref}[18]$. In the collisionless limit $\left(\nu_{* a} \rightarrow 0\right)$ the velocity integrals can be evaluated analytically, giving for the bracketed term,

$$
\begin{gathered}
{\left[\nu_{D}^{a} \tau_{a \Omega}\right]=\frac{3 \sqrt{\pi}}{4} \sum_{b} \frac{n_{b} Z_{b}^{2}}{n_{a} Z_{a}^{2}}\left[\sqrt{1+x_{a b}^{2}}+x_{a b}^{2} \ln \left(\frac{x_{a b}}{1+\sqrt{1+x_{a b}^{2}}}\right)\right]} \\
{\left[x_{a}^{2} \nu_{D}^{a} \tau_{a a}\right]=\frac{3 \sqrt{\pi}}{4} \sum_{b} \frac{n_{b} Z_{b}^{2}}{n_{a} Z_{a}^{2}} \frac{1}{\sqrt{1+x_{a b}^{2}}}} \\
{\left[x_{a}^{4} \nu_{D}^{a} \tau_{a a}\right]=\frac{3 \sqrt{\pi}}{4} \sum_{b} \frac{n_{b} Z_{b}^{2}}{n_{a} Z_{a}^{2}}\left[\frac{2\left(1+\frac{5}{4} x_{a b}^{2}\right)}{\left(1+x_{a b}^{2}\right)^{\frac{3}{2}}}\right] .}
\end{gathered}
$$

These can be used directly to derive the single ion expression for the collisionless bootstrap current. $x_{a b}$ is defined as $v_{t b} / v_{t a}$. 


\section{Appendix C: FRICTION COEFFICIENTS}

The friction coefficients are defined by

$$
l_{i j}^{a b}=\left(\sum_{k} \frac{n_{a} m_{a}}{\tau_{a k}} M_{a k}^{i-1, j-1}\right) \delta_{a b}+\frac{n_{a} m_{a}}{\tau_{a b}} N_{a b}^{i-1, j-1}
$$

where $n_{a}$ is the particle density, $m_{a}$ is the particle mass, and $\tau_{a b}$ is the collision time for species a. $M_{a b}$ and $N_{a b}$ are given below.

$$
\begin{aligned}
& M_{a b}^{00}=-\left(1+\frac{m_{a}}{m_{b}}\right)\left(1+x_{a b}^{2}\right)^{-\frac{3}{2}} \\
& N_{a b}^{00}=\left(1+\frac{m_{a}}{m_{b}}\right)\left(1+x_{a b}^{2}\right)^{-\frac{3}{2}} \quad=\quad-M_{a b}^{00} \\
& M_{a b}^{01}=-\frac{3}{2}\left(1+\frac{m_{a}}{m_{b}}\right)\left(1+x_{a b}^{2}\right)^{-\frac{5}{2}} \\
& M_{a b}^{10}=M_{a b}^{01} \\
& N_{a b}^{01}=\quad \frac{T_{a}}{T_{b}} \frac{v_{a b}}{v_{t b}} N_{b a}^{10}=-\frac{T_{a}}{T_{b}} M_{b a}^{10} \\
& N_{a b}^{10}=-M_{a b}^{10} \\
& =\frac{3}{2}\left(1+\frac{m_{a}}{m_{b}}\right)\left(1+x_{a b}^{2}\right)^{-\frac{5}{2}} \\
& M_{a b}^{11}=-\left(\frac{13}{4}+4 x_{a b}^{2}+\frac{15}{2} x_{a b}^{4}\right)\left(1+x_{a b}^{2}\right)^{-\frac{5}{2}} \\
& N_{a b}^{11}=\quad \frac{27}{4} \frac{T_{a}}{T_{b}} x_{a b}^{2}\left(1+x_{a b}^{2}\right)^{-\frac{5}{2}}
\end{aligned}
$$

In the expression for the friction coefficients the summation is over all species, including species a, however, the delta function requires that the summation only be evaluated when $\mathrm{a}=\mathrm{b}$. The quantity $x_{a b}$ is the ratio of thermal velocities $v_{t b} / v_{t a}$. There are symmetry relations that help to understand these coefficients and their relationships. The selfadjointness of the Coulomb collision operator gives,

$$
\begin{aligned}
M_{a b}^{i j} & =M_{a b}^{j i} \\
\frac{N_{a b}^{i j}}{T_{a} v_{t a}} & =\frac{N_{b a}^{j i}}{T_{b} v_{t b}}
\end{aligned}
$$

which imply that $l_{i j}^{a b}=l_{j i}^{b a}$, and from momentum conservation,

$$
M_{a b}^{j 0}=-N_{a b}^{j 0}
$$

There are additional terms in the expression for the friction forces that correspond to higher order flows. These higher order flows must be expressed in terms of the fluid and heat flows in order to be useful. This is done in detail in Ref[15]. 


\section{ACKNOWLEDGMENTS}

The author is grateful to S. Jardin for suggestions and guidance during the work. The author would like to acknowledge useful discussions with D. Ehst, S. Hirshman, and T. K. Mau. This work was supported by the US DoE Contract No. DE-AC02-76-CHO-3037. 


\section{References}

[1] R. J. BICKERTON, J. W. CONNOR, and J. B. TAYLOR, Nature Phys. Sci., 229 (1971) 110

[2] M. C. ZARnSTORFF, M. G. BELL, M. BITTER, et al., Phys. Rev. Lett., 60 (1988) 1306.

[3] J. G. CORDEY, C. D. CHALlis, and P. M. STUBBERFIELD, Plasma Phys., 80 (1988) 1625.

[4] M. KIKUCHI, M. AZUMI, S. TSUJI, et al., Nucl. Fusion, 30 (1990) 343.

[5] M. N. ROSEnbluth, R. D. HAZEltine, and F. L. Hinton, Phys. Fluids, 15 (1972) 116.

[6] R. D. hazeltine, F. L. Hinton, and M. N. ROSEnBluth, Phys. Fluids, 18 (1973) 1645.

[7] F. L. HiNTON and M. N. ROSENBLUTh, Phys. Fluids, 16 (1973) 836.

[8] F. L. HiNTON and R. D. HAZELTiNe, Rev. Modern Phys., 48 (1976) 239.

[9] D. J. SIGMAR and P. H. RUTHERFORD, Nucl. Fusion, 13 (1973) 677.

[10] D. J. SIGMAR, Nucl. Fusion, 13 (1973) 17.

[11] F. L. HINTON and T. B. MOORE, Nucl. Fusion, 14 (1974) 639.

[12] S. P. HIRShMAN, D. J. SIGMAR, and J. F. ClARKE, Phys. Fluids, 19 (1976) 656.

[13] S. P. HIRSHMAN and D. J. SIGMAR, Phys. Fluids, 19 (1976) 1532.

[14] S. P. HIRSHMAN and D. J. SIGMAR, Phys. Fluids, 20 (1977) 418.

[15] S. P. HIRSHMAN, Phys. Fluids, 20 (1977) 589.

[16] S. P. HIRSHMAN, Phys. Fluids, 21 (1978) 224.

[17] S. P. HIRSHMAN, Phys. Fluids, 21 (1978) 1295.

[18] S. P. HIRSHMAN and D. J. SIGMAR, Nucl. Fusion, 21 (1981) 1079.

[19] S. P. HIRSHMAN, R. J. HAWRYLUK, and B. BIRGE, Nucl. Fusion, 17 (1977) 611.

[20] S. P. HIRSHMAN, Phys. Fluids, 31 (1988) 3150.

[21] H. R. WILSON, Nucl. Fusion, 32 (1992) 257.

[22] G. R. HARRIS, "Comparisons of Different Bootstrap Current Expressions", Department de Recherches sur la Fusion Controlee Report, EUR-CEA-FC-1436 (November 1991). 
[23] N. POMPHREY, "Bootstrap Dependence on Plasma Profile Parameters", Princeton Plasma Physics Laboratory Report, PPPL-2854 (August 1992).

[24] K. LACKNER, "Maximum Bootstrap Current Compatible with Ballooning-Stable Pressure Profiles", Max-Planck Institute for Plasma Physics Report, IPP 5/17 (November 1987).

[25] F. NAJMABADI, R. W. CONN, and the ARIES team, "The ARIES-I Tokamak Reactor Study, Final Report, 1991", University of California Los Angeles Report, UCLA-PPG-1323 (1991).

[26] J. DELUCIA, S. C. JARDIN, and A. M. M. TODD, J. Comp. Physics 37 (1980) 183.

[27] C. S. CHANG, "Bootstrap Current Driven by Alpha Particles in a Fusion Reacting Tokamak", in Proc. of the 19th European Conference on Cont. Fusion and Plasma Physics, Innsbruck (1992) p. 1-315.

[28] C. T. HSU, K. C. SHAING, R. P. GORMLY, et al., Phys. Fluids B, 4 (1992) 4023.

[29] H. WOBIG, "On Bootstrap Current in Toroidal Systems", Max-Planck Institute for Plasma Physics Report, IPP 2/297 (October 1988).

[30] M. N. ROSENBLUTH and P. H. RUThERFORD, "Tokamak Plasma Stability", in Fusion, ed. E. TELLER, Academic Press (1981).

[31] K. T. TSANG and J. D. CALLEN, Phys. Fluids, 19 (1976) 667.

[32] S. P. HIRSHMAN and S. C. JARDIN, Phys. Fluids, 22 (1979) 741.

[33] A. D. TURnbull, M. A. SECREtan, F. Troyon, et al., J. Comput. Phys., 66 (1986) 391.

[34] J. MANICKAM, J. Comput. Phys., 66 (1986) 324.

[35] W. HOWL, D. TURnbull, T. S. TAYlor, et al., Phys. Fluids B, 4 (1992) 1724.

[36] C. KESSEL, J. MANICKAM, G. REWOLDT, et al., Phys. Rev. Lett., to be published. 
Table 1: Summary of Results Presented in Figures

\begin{tabular}{|c|c|c|c|c|c|c|c|c|c|}
\hline figure & $\mu_{o} p_{o}$ & $a_{p}$ & $b_{p}$ & $T_{o}(k e V)$ & $a_{T}$ & $b_{T}$ & $\frac{T_{o}}{\langle T\rangle}$ & $\frac{n_{o}}{\langle n}$ & $\frac{T_{b s}}{I_{p}}$ \\
\hline $\begin{array}{c}\text { Fig. 1a } \\
\text { H-S }\end{array}$ & 2.40 & 2.00 & 1.00 & 30.0 & 1.75 & 1.00 & & & \\
Har & & & & & & & & & 0.91 \\
Hir & & & & & & & & & 0.74 \\
Fig. 1b & 2.40 & 2.00 & 1.00 & 15.0 & 1.75 & 1.00 & & & \\
H-S & & & & & & & & & 0.77 \\
Har & & & & & & & & & 0.58 \\
Fig. 1c & 2.40 & 2.00 & 1.00 & 7.5 & 1.75 & 1.00 & & & \\
H-S & & & & & & & & & 0.54 \\
Har & & & & & & & & & 0.25 \\
\hline Fig. 2 & 2.40 & 2.00 & 1.00 & 30.0 & 1.75 & 1.00 & & & \\
eqn(11) (a) & & & & & & & & & 0.91 \\
eqn(14) (b) & & & & & & & & & 0.84 \\
eqn(13) (c) & & & & & & & & & 1.07 \\
\hline Fig. 3 & & 2.00 & 1.00 & & 1.50 & 1.00 & & & \\
(a) & 0.76 & & & 19.0 & & & & & 0.32 \\
(b) & 1.52 & & & 38.0 & & & & & 0.64 \\
(c) & 2.25 & & & 57.0 & & & & & 0.94 \\
(d) & 3.00 & & & 76.0 & & & & & 1.22 \\
\hline Fig. 4 & 1.20 & & & 30.0 & & & & & \\
(a) & & 3.00 & 1.00 & & 2.00 & 1.00 & & & \\
(b) & & 1.50 & 1.00 & & 1.25 & 1.00 & & & \\
(c) & & 2.00 & 1.50 & & 1.75 & 1.50 & & & \\
(d) & & 2.00 & 4.00 & & 1.75 & 4.00 & & & \\
\hline Fig. 5 & 1.20 & 2.00 & 1.00 & 30.0 & & 1.00 & & & \\
(a) & & & & & 0.50 & & 1.62 & 2.92 & 0.69 \\
(b) & & & & & 1.00 & & 2.27 & 2.27 & 0.60 \\
(c) & & & & & 1.50 & & 2.92 & 1.62 & 0.50 \\
(d) & & & & & 1.95 & & 3.50 & 1.06 & 0.42 \\
\hline Fig. 6 & 1.20 & 2.00 & 1.00 & & 1.50 & 1.00 & & & \\
(a) & & & & 30.0 & & & & & 0.50 \\
(b) & & & & 7.50 & & & & & 0.40 \\
(c) & & & & 3.75 & & & & & 0.16 \\
\hline
\end{tabular}




\section{Figure Captions}

FIG. 1. The bootstrap current profiles and the equilibrium current profile as a function of poloidal flux $\hat{\psi}$ for a high pressure equilibrium for the Hirshman-Sigmar model, the Harris model, and the Hirshman model at $30 \mathrm{keV}$ (a), $15 \mathrm{keV}$ (b), and $7.5 \mathrm{keV}$ (c).

FIG. 2. The bootstrap current profiles and the equilibrium current profile as a function of poloidal flux $\hat{\psi}$ for a high pressure equilibrium for the full integral trapped particle fraction (a), Eqn.(14) (b), and Eqn.(13) (c).

FIG. 3. The bootstrap current profiles and the equilibrium current profile as a function of poloidal flux $\hat{\psi}$ for $\beta_{p}$ values of 1.0 (a), 2.0 (b), 3.0 (c), and 4.0 (d).

FIG. 4. The bootstrap current profiles and the equilibrium current profile as a function of poloidal flux $\hat{\psi}$ for various pressure profiles showing that the shape of the bootstrap current profile shape is determined by the location of the pressure gradient. Table 1 shows the plasma profile parameters for the cases (a)-(d).

FIG. 5. The bootstrap current profiles and the equilibrium current profile as a function of poloidal flux $\hat{\psi}$, for various density profiles showing that peaked density profiles lead to larger bootstrap current and broad ones to less bootstrap current. Table 1 shows the plasma profile parameters for cases (a)-(d).

FIG. 6. The bootstrap current profiles and the equilibrium current profile as a function of the poloidal flux $\hat{\psi}$ for various peak temperature values to show the reduction in the bootstrap current as the collisionality is increased. The peak temperatures are $30 \mathrm{keV}(\mathrm{a})$, $7.5 \mathrm{keV}$ (b), and $3.75 \mathrm{keV}$ (c).

FIG. 7. An equilibrium with a non-monotonic safety factor profile that is typical of the sort of equilibria that would be generated by a nearly $100 \%$ bootstrap driven tokamak. The pressure (a), safety factor (b), parallel current density (c), temperature (d), and density (e) profiles as a function of poloidal flux $\hat{\psi}$. The equilibrium is created by making the current profile almost perfectly align with the bootstrap current profile.

FIG. 8. The bootstrap current profile ("B") and the equilibrium current profile as a function of the poloidal flux $\hat{\psi}$ when trying to align the bootstrap with the equilibrium profile. The temperature and density curves show the actual profile assumed and the desired profile (" $\mathrm{B}$ ") from inverting the collisionless bootstrap equation. 


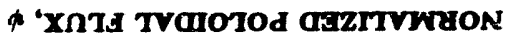

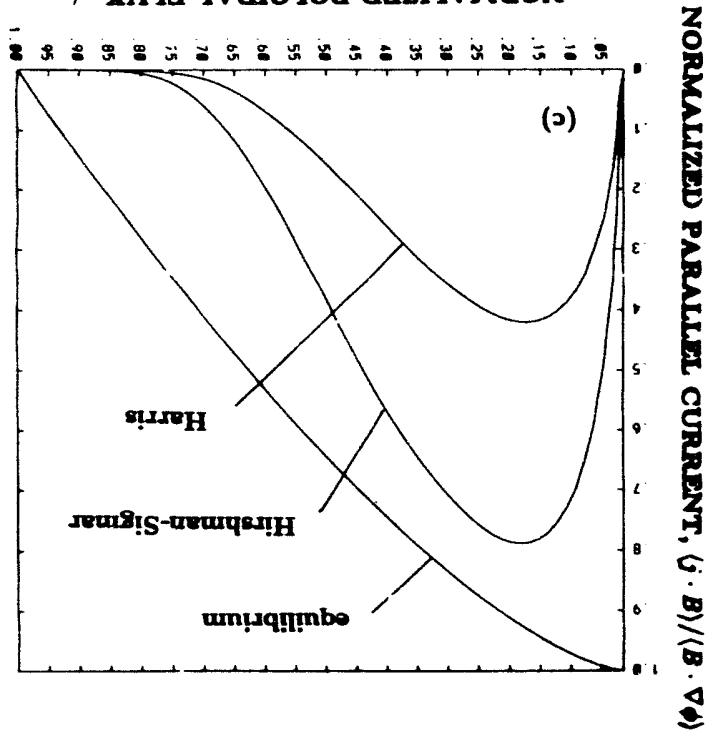

* 'XnTi Tranotod aazitywyon

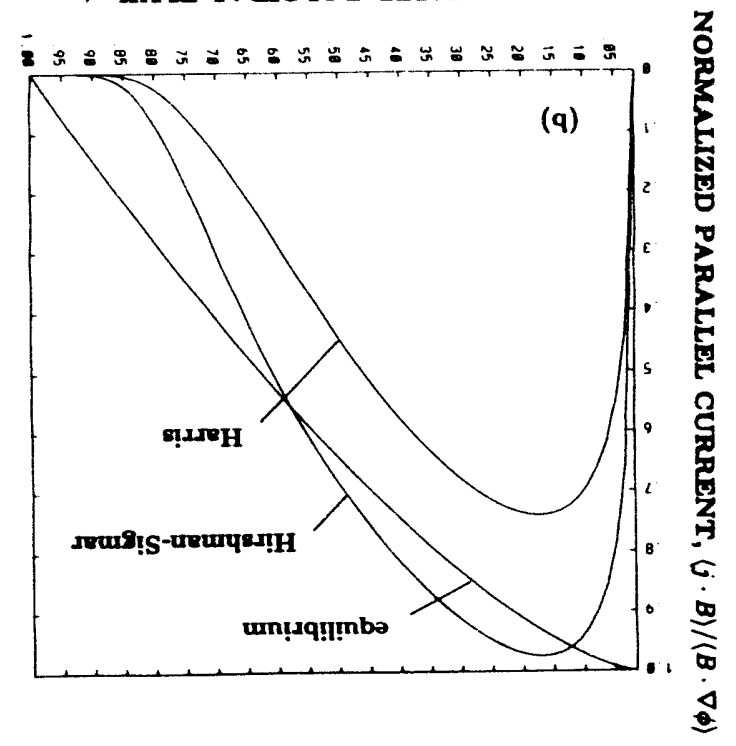

中 'xnti traiotod atzitvirion

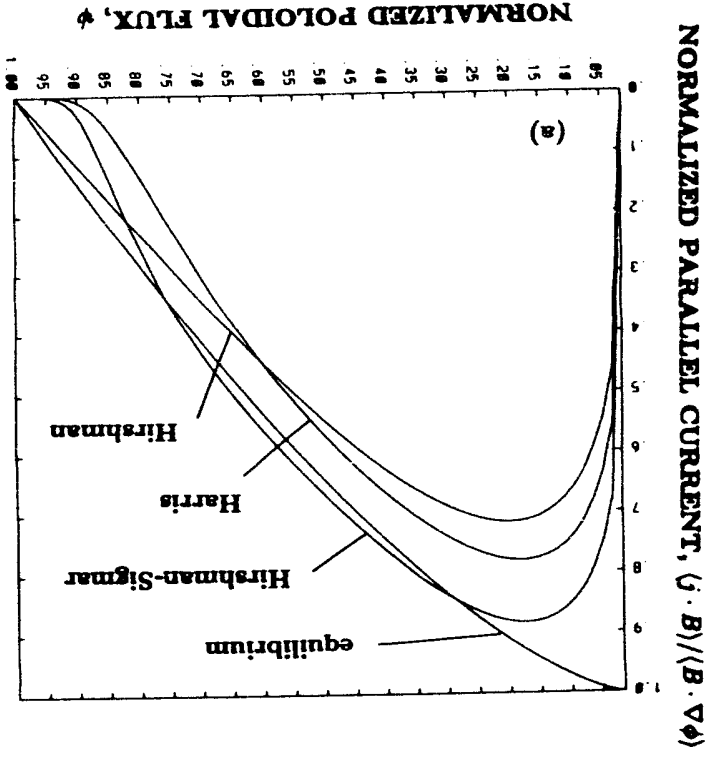




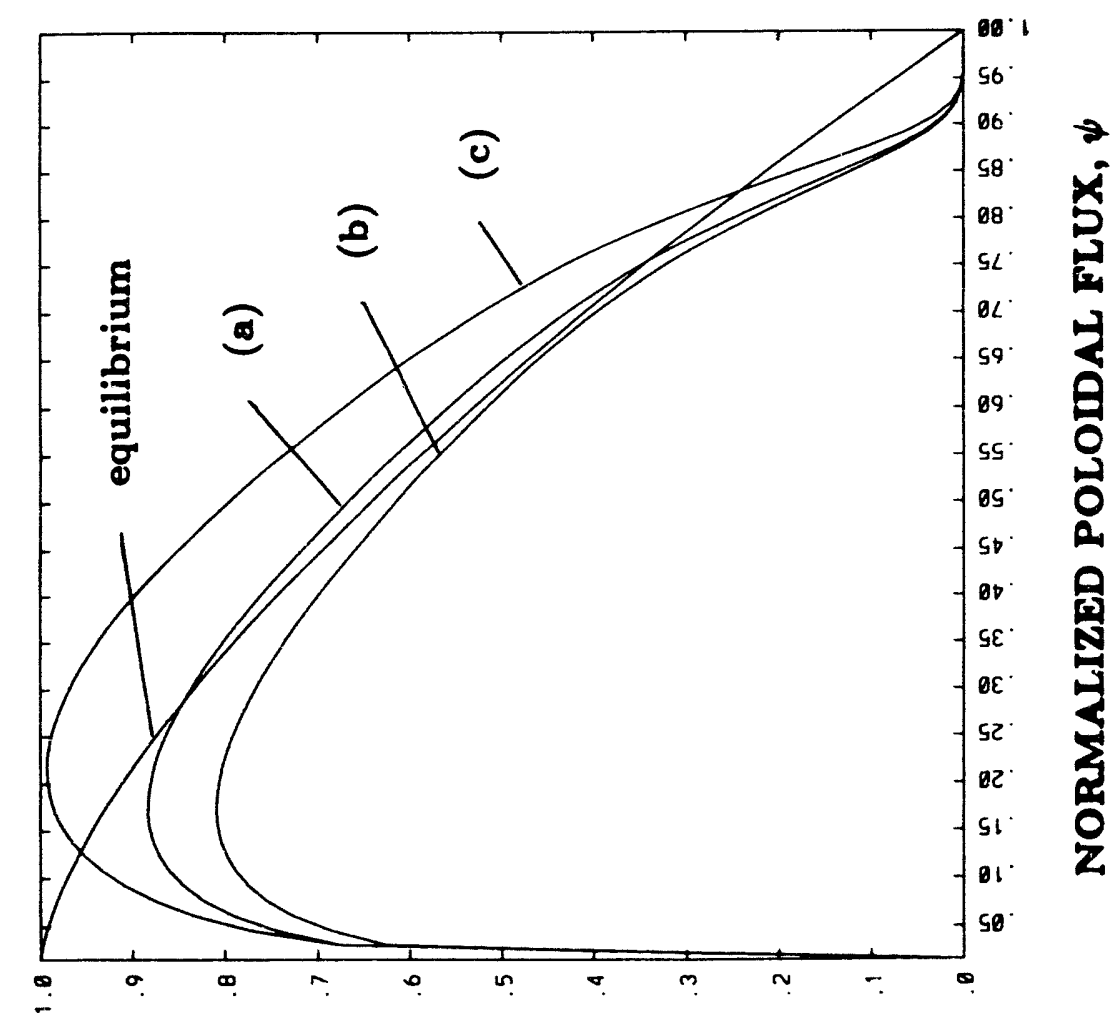

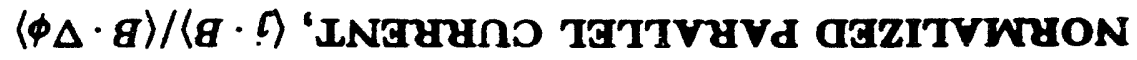




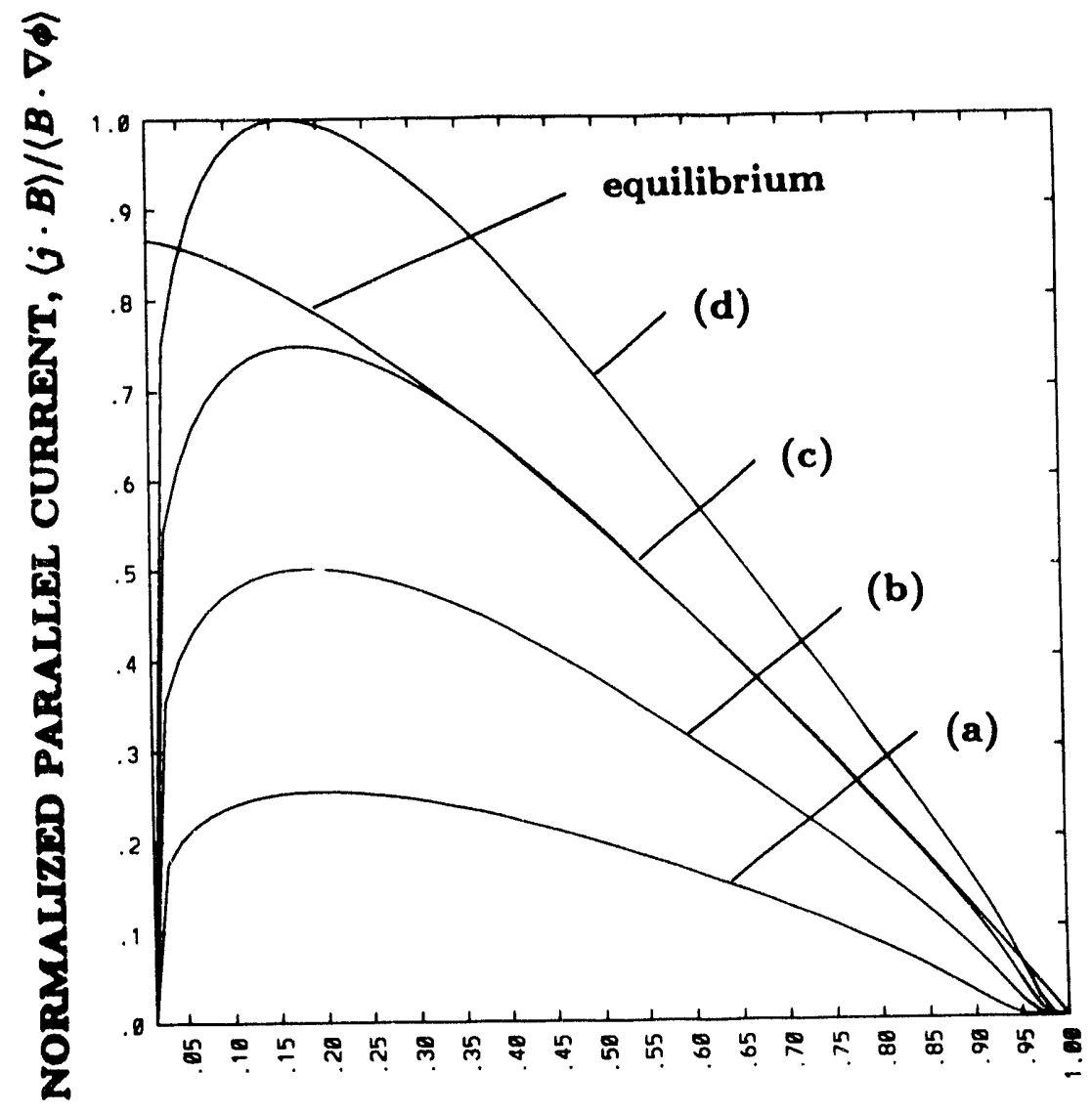

NORMALIZWD POLOIDAL FLUX, $\psi$ 

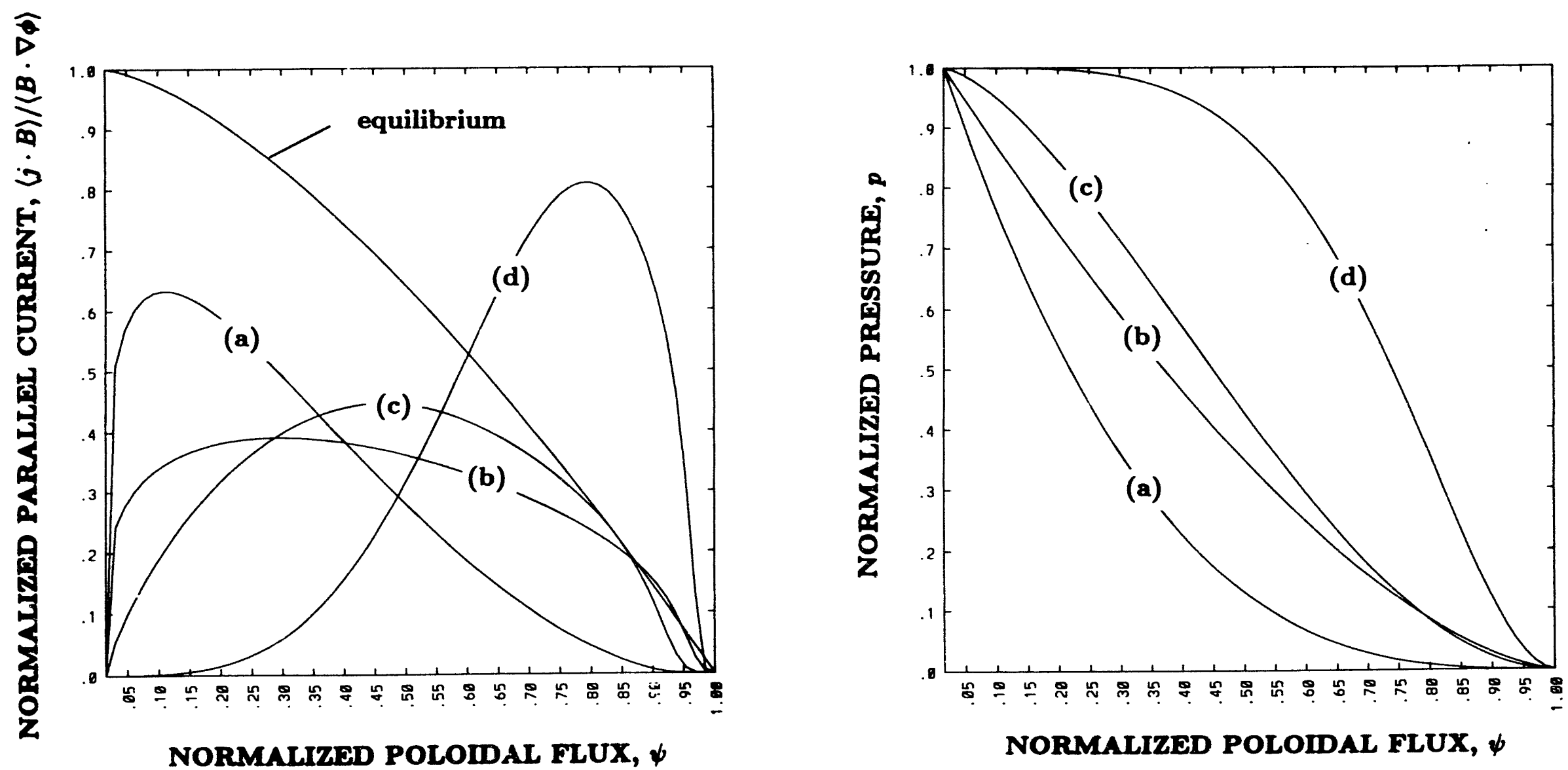

๑ొ 

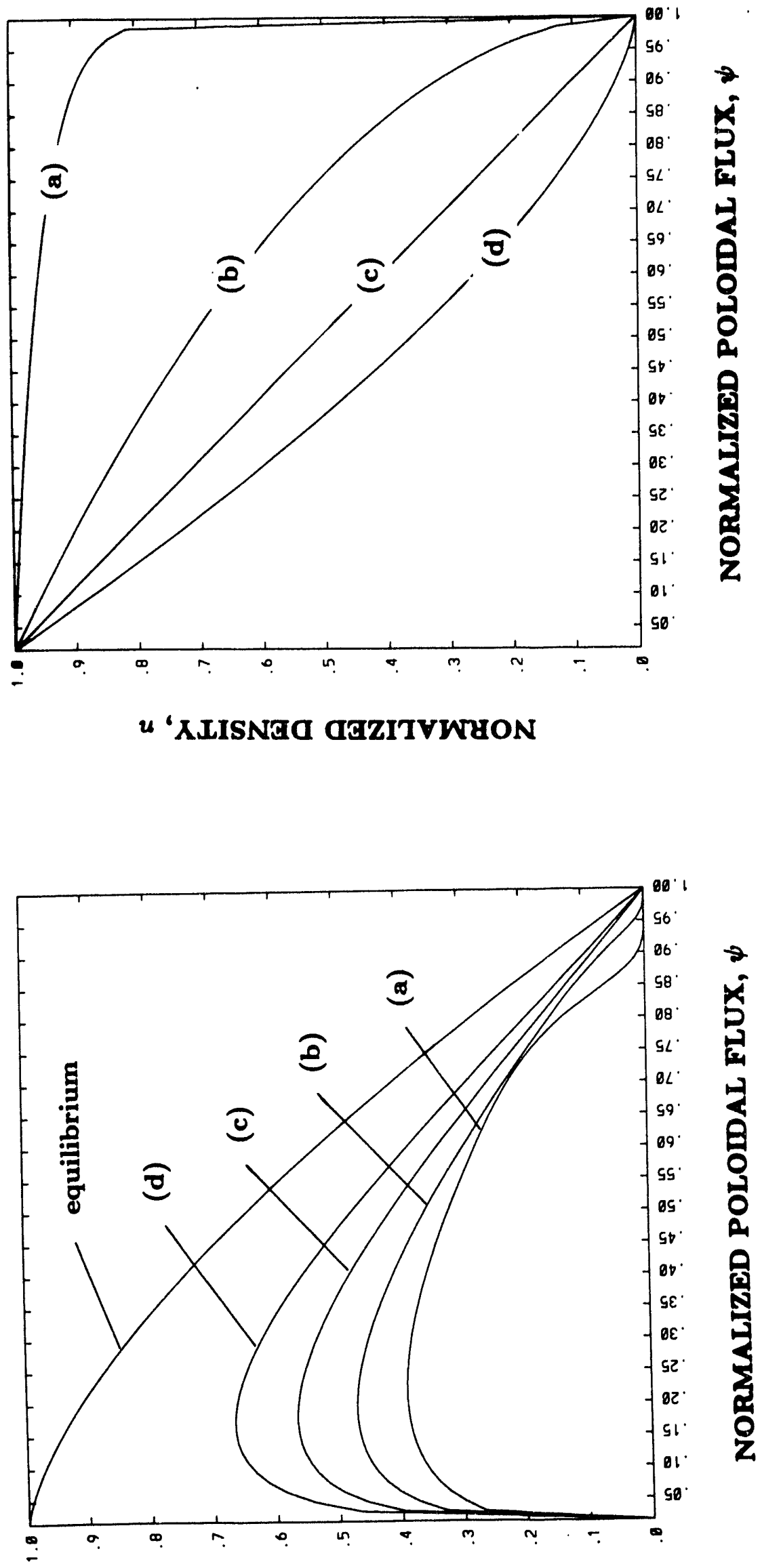

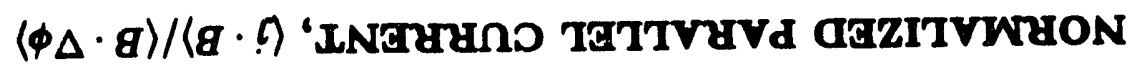


9 อ.กริ!.

\section{"X 'XIT TVaIOTOd aGzITVHYON}

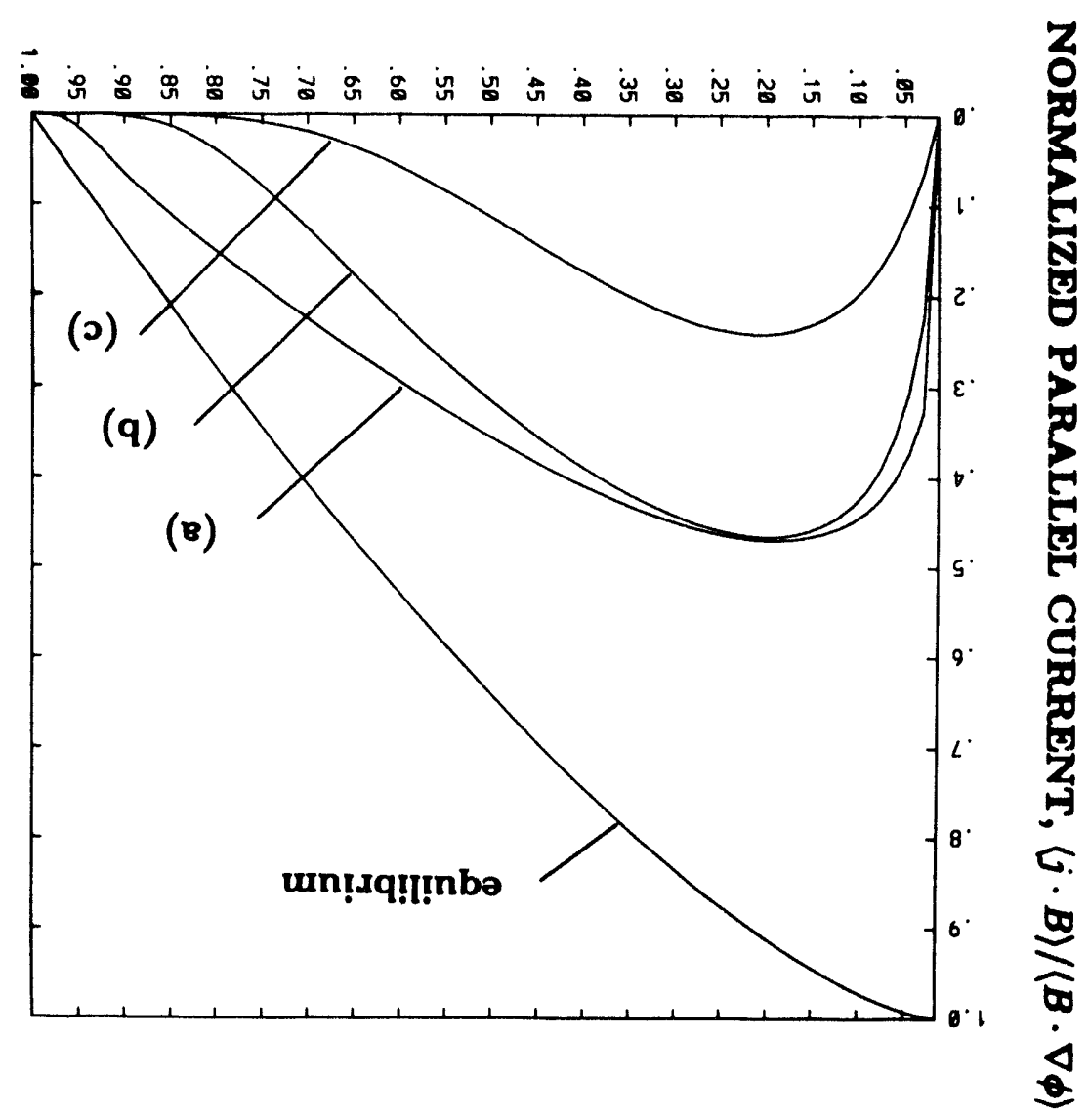




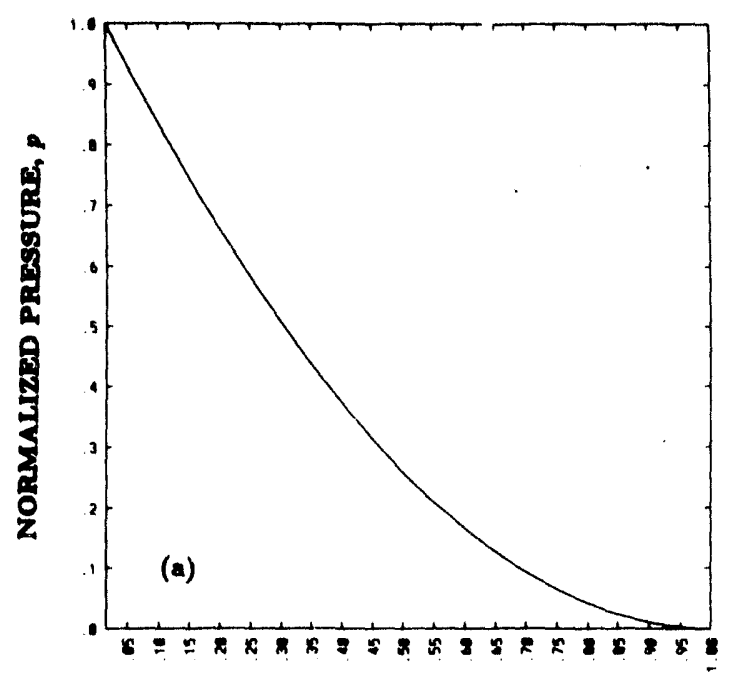

NORMALIZED POLOEAL FLUX, $\psi$

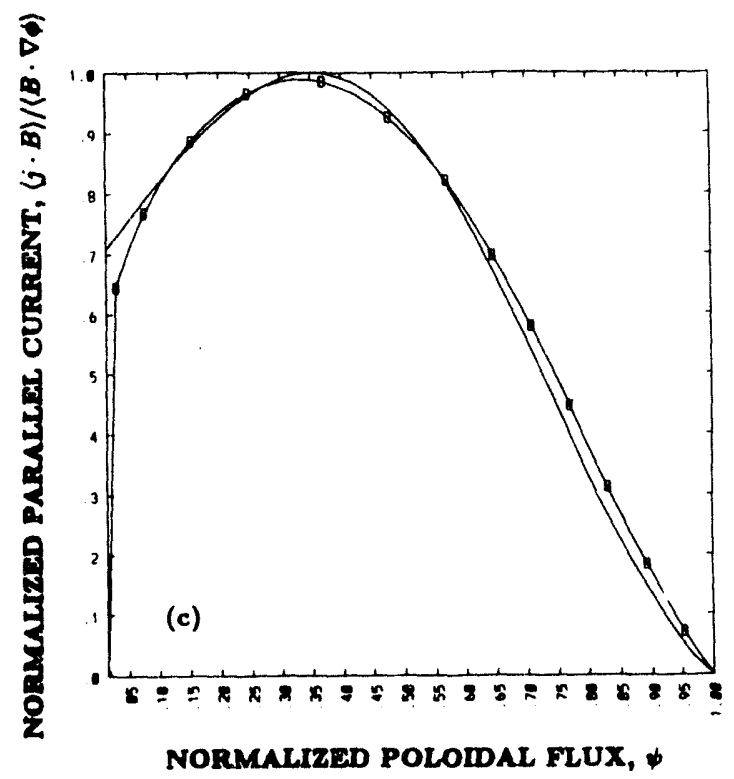

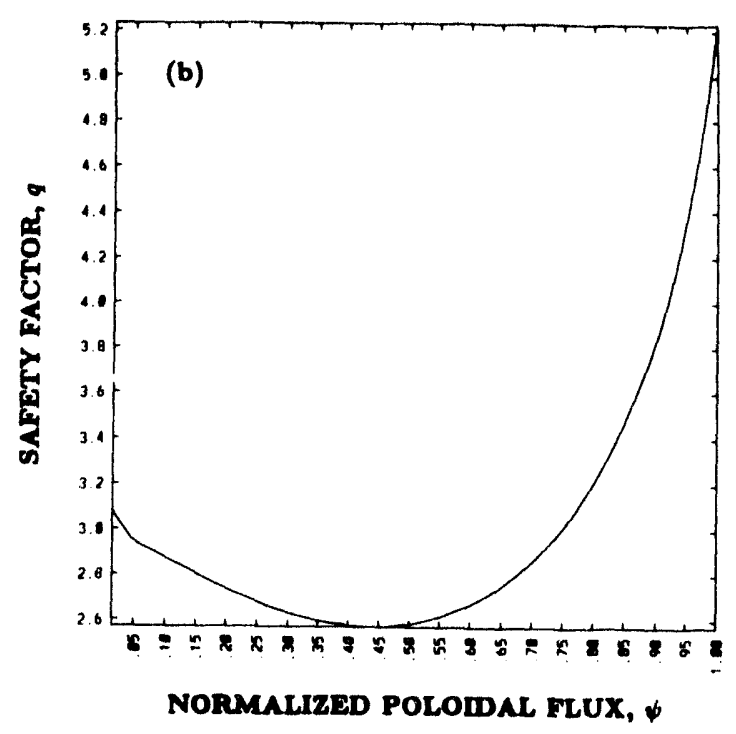

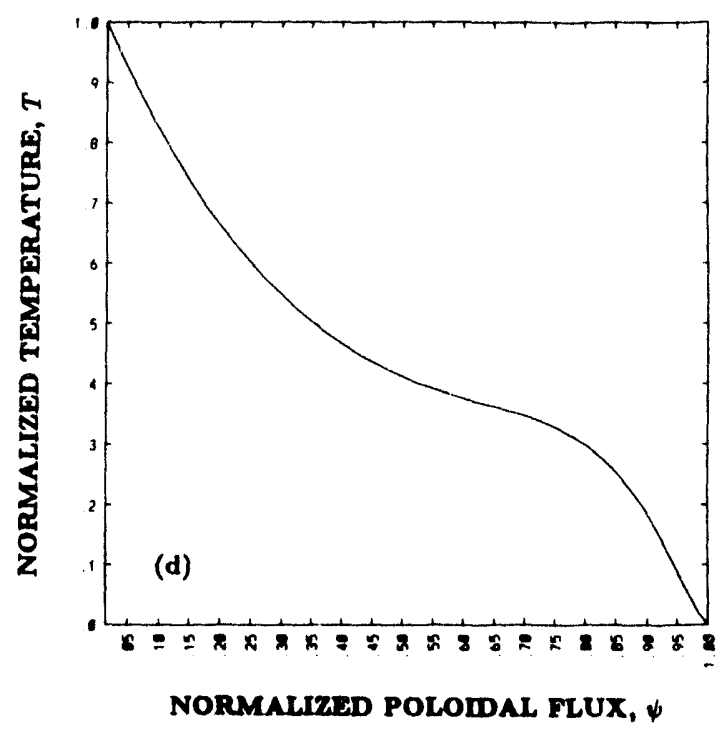

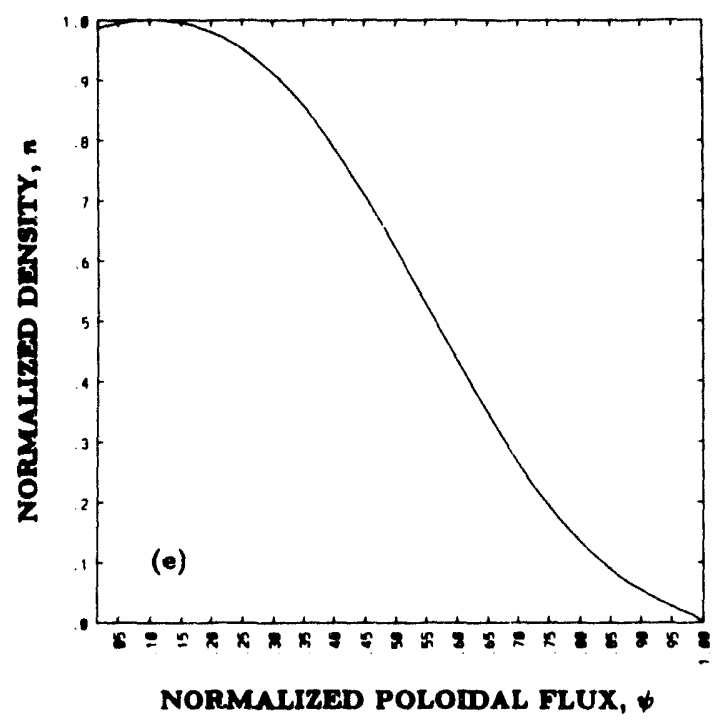



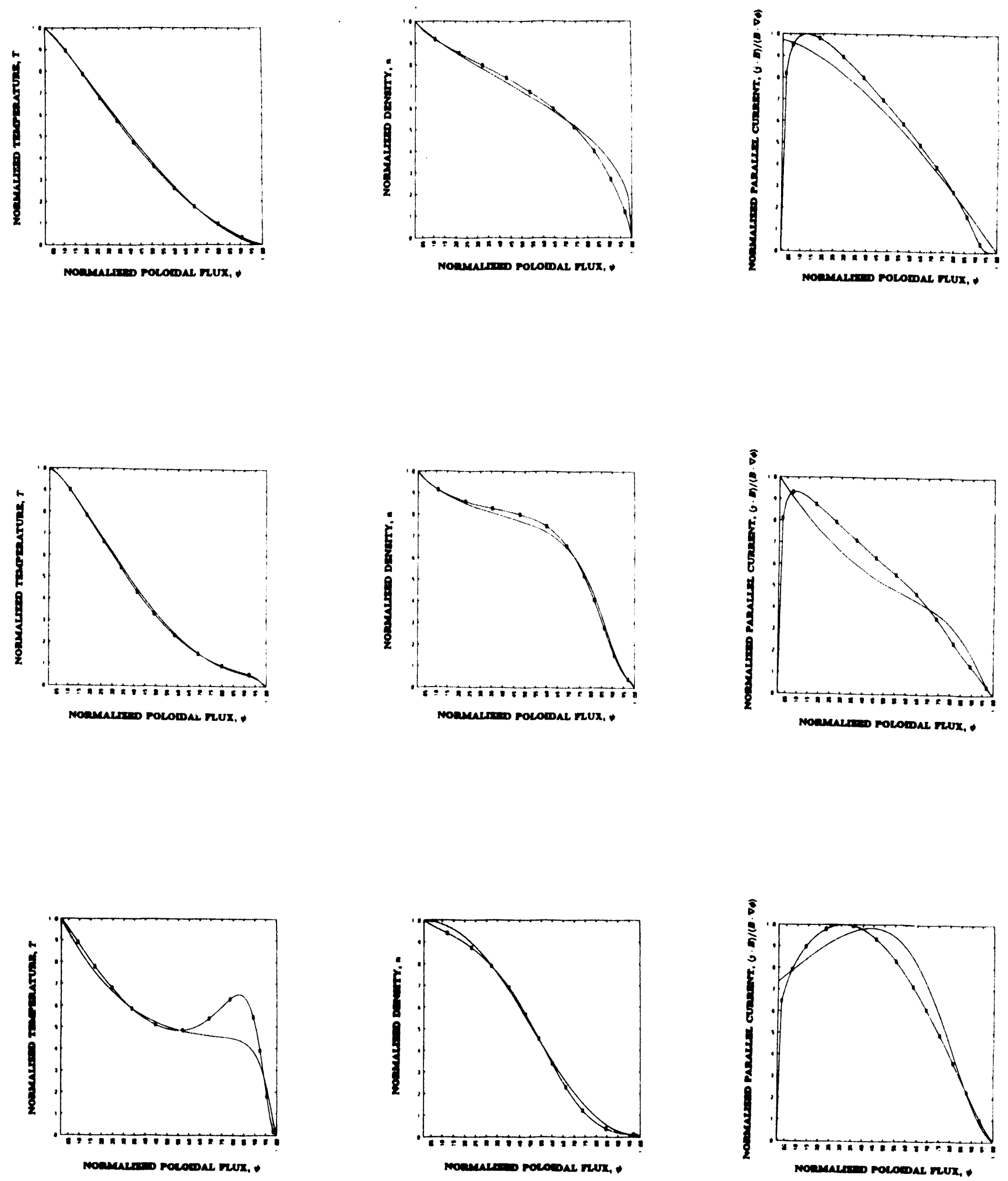

Figure 8 
Dr. F. Paoloni, Univ. of Wollongong, AUSTRALIA

Prof. M.H. Brennan, Univ. of Sydney, AUSTRALIA

Plasma Research Lab., Australian Nat. Univ., AUSTRALIA

Prof. I.R. Jones, Flinders Univ, AUSTRALIA

Prot. F. Cap, Inst. for Theoretical Physics, AUSTRIA

Prof. M. Heindler, Institut für Theoretische Physik, AUSTRIA

Prof. M. Goossens, Astronomisch Instituut, BELGIUM

Ecole Royale Militaire, Lab. de Phy. Plasmas, BELGIUM

Commission-European, DG. XII-Fusion Prog., BELGIUM

Prof. R. Bouciqué, Rijksuniversiteit Gent, BELGIUM

Dr. P.H. Sakanaka, Instituto Fisica, BRAZIL

Prot. Dr. I.C. Nascimento, Instituto Fisica, Sao Paulo, BRAZIL Instituto Nacional De Pesquisas Espaciais-INPE, BRAZIL

Documents Otfice, Alomic Energy of Canada Lid., CANADA

Ms. M. Morin, CCFMTokamak de Varennes, CANADA

Dr. M.P. Bachynski, MPB Technologies, Inc., CANADA

Dr. H.M. Skarsgard, Univ. of Saskatchewan, CANADA

Prol. J. Teichmann, Univ. of Montreal, CANADA

Prof. S.R. Sreenivasan, Univ. of Calgary, CANADA

Prol. T.W. Johnston, INRS-Energie, CANADA

Dr. R. Bolton, Centre canadien de fusion magnétique, CANADA

Dr. C.R. James., Univ. of Alberta, CANADA

Dr. P. Lukác, Komenského Universzita, CZECHO-SLOVAKIA

The Librarian, Culham Laboratory, ENGLAND

Library, R61, Ruthertord Appleton Laboratory, ENGLAND

Mrs. S.A. Hutchinson, JET Library, ENGLAND

Dr. S.C. Sharma, Univ. of South Pacific, FIJI ISLANDS

P. Mähönen, Univ. of Helsinki, FINLAND

Prof. M.N. Bussac, Ecole Polytechnique., FRANCE

C. Mouttet, Lab. de Physique des Milieux lonisés, FRANCE

J. Radet, CEN/CADARACHE - Bat 506, FRANCE

Prot. E. Economou, Univ. of Crete, GREECE

Ms. C. Rinni, Univ. of loannina, GREECE

Preprint Library. Hungarian Academy of Sci., HUNGARY

Dr. B. DasGupta, Saha Inst. of Nuclear Physics, INDIA

Dr. P. Kaw, Inst. for Plasma Research, INDIA

Dr. P. Rosenau, Israel Inst. of Technology, ISRAEL

Librarian, International Center for Theo Physics, ITALY

Miss C. De Palo, Associazione EURATOM-ENEA, ITALY

Dr. G. Grosso, Istituto di Fisica del Plasma, ITALY

Prof. G. Rostangni, Istituto Gas lonizzati Del Cnr, ITALY
Dr. H. Yamato, Toshiba Res \& Devel Center, JAPAN

Prof. I. Kawakami, Hiroshima Univ., JAPAN

Prof. K. Nishikawa, Hiroshima Univ., JAPAN

Librarian, Naka Fusion Research Establishment, JAERI, JAPAN

Director, Japan Atomic Energy Research Inst., JAPAN

Prof. S. Itoh, Kyushu Univ., JAPAN

Research Into. Ctr., National Instit. for Fusion Science, JAPAN

Prof. S. Tanaka, Kyoto Univ., JAPAN

Library, Kyoto Univ., JAPAN

Prof. N. Inoue, Univ. of Tokyo, JAPAN

Secretary, Plasma Section, Electrotectinical Lab., JAPAN

S. Mori, Technical Advisor, JAERI, JAPAN

Dr. O. Mitarai, Kumamoto Inst. of Technology, JAPAN

Dr. G.S. Lee, Korea Basic Sci. Ctr., KOREA

J. Hyeon-Sook, Korea Atomic Energy Research Inst., KOREA

D.I. Choi, The Korea Adv. Inst. of Sci. \& Tech., KOREA

Prof. B.S. Liley, Univ. of Waikato, NEW ZEALAND

Inst of Physics, Chinese Acad Sci PEOPLE'S REP. OF CHINA

Library, Inst. of Plasma Physics, PEOPLE'S REP. OF CHINA

Tsinghua Univ. Library, PEOPLE'S REPUBLIC OF CHINA

Z. Li, S.W. Inst Physics, PEOPLE'S REPUBLIC OF CHINA

Prot. J.A.C. Cabral, Instituto Superior Tecnico, PORTUGAL

Prof. M.A. Hellberg, Univ of Natal, S. AFRICA

Prof. D.E. Kim, Pohang Inst. of Sci. \& Tech., SO. KOREA

Prof. C.I.E.M.A.T, Fusion Division Library, SPAIN

Dr. L. Stenflo, Univ. of UMEA, SWEDEN

Library, Royal Inst. of Technology, SWEDEN

Prof. H. Wilhelmson, Chalmers Univ, of Tech., SWEDEN

Centre Phys. Des Plasmas, Ecole Polytech, SWITZERLAND

Bibliotheek, Inst. Voor Plasma-Fysica, THE NETHERLANDS

Asst. Prol. Dr. S. Cakir, Middle East Tech. Univ., TURKEY

Dr. V.A. Glukhikh,Sci. Res. Inst. Electrophys.I Apparatus, USSR

Dr. D.D. Ryutov, Siberian Branch of Academy of Sci., USSR

Dr. G.A. Eliseev, I.V. Kurchatov Inst., USSR

Libranian, The Ukr.SSR Academy of Sciences, USSR

Dr. L.M. Kovrizhnykh, Inst. of General Physics, USSR

Kemforschungsanlage $\mathrm{GmbH}$, Zentralbibliothek, W. GERMANY

Bibliothek, Inst. Für Plasmaforschung, W. GERMANY

Prof. K. Schiridler, Ruhr-Universitát Bochum, W. GERMANY

Dr. F. Wagner, (ASDEX), Max-Planck-Institut, W. GERMANY

Librarian, Max-Planck-Institut, W. GERMANY 

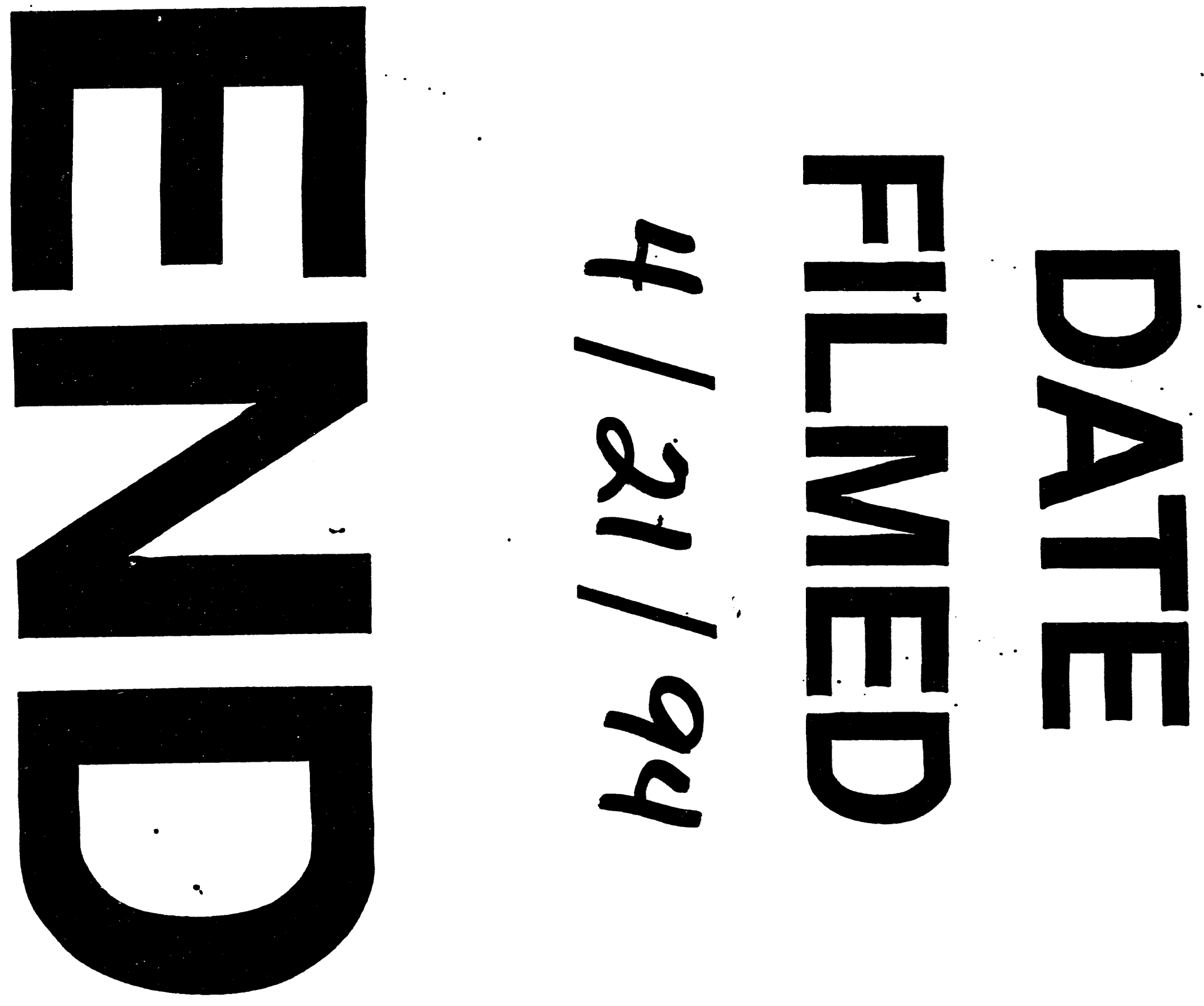
